\title{
Evolutionary and functional patterns of shared gene neighborhood in fungi
}

Marina Marcet-Houben ${ }^{1,2}$ and Toni Gabaldón ${ }^{1,2,3, *}$

${ }^{1}$ Centre for Genomic Regulation (CRG), The Barcelona Institute of Science and Technology, Dr. Aiguader 88, Barcelona 08003, Spain

${ }^{2}$ Universitat Pompeu Fabra (UPF). 08003 Barcelona, Spain.

${ }^{3}$ ICREA, Pg. Lluís Companys 23, 08010 Barcelona, Spain.

* Author for correspondence: toni.gabaldon.bcn@gmail.com

\begin{abstract}
Gene clusters comprise genomically co-localized and potentially co-regulated genes that tend to be conserved across species. In eukaryotes, multiple examples of metabolic gene clusters are known, particularly among fungi and plants. However, little is known about how gene clustering patterns vary among taxa, or among functional roles. Furthermore, mechanisms for the formation, maintenance and evolution of gene clusters remain a puzzle. We surveyed 341 fungal genomes to discover gene clusters shared by different species, independently of their functions. We inferred 12,120 cluster families, which comprised roughly one third of the gene space, and were enriched in diverse cellular functions. Additionally, most clusters did not encode transcription factors, suggesting they are regulated distally. We used phylogenomics to characterize the evolutionary history of these clusters. We found that most clusters originated once, and were transmitted vertically, coupled to differential loss. However, convergent
\end{abstract}


evolution -i.e. independent appearance of the same cluster- is more prevalent than anticipated. Finally, horizontal gene transfer of entire clusters was rather restricted, with the exception of secondary metabolism clusters. Altogether our results provide new insights on the evolution of gene clustering as well as a broad catalogue of evolutionarily conserved gene clusters whose function remains to be elucidated.

\section{Introduction}

Chromosomal rearrangements continuously shuffle the ordering of genes along a genome. Evolution of gene order has been extensively studied in prokaryotes, establishing a link between evolutionary conserved neighborhood and functional relatedness ${ }^{1}$. In eukaryotes a similar (albeit much weaker) trend has been observed, with the observation that certain groups of genes tend to remain in close proximity across large evolutionary distances, which suggests the action of selection ${ }^{2,3}$.

Conservation of gene proximity in eukaryotes has been associated to genes that share similar expression patterns ${ }^{4-7}$, are part of the same protein-protein interaction network ${ }^{8,9}$, or are functionally related ${ }^{10,11}$. Examples of clusters of functionally related genes include those involved in nutrient or nitrogen utilization ${ }^{12-15}$ or those responsible for the synthesis of small secondary metabolites such as antibiotics, toxins and pigments ${ }^{16-18}$. In recent years, extensive research has focused on the detection of this last category, as it has clinical and industrial relevance.

From an evolutionary perspective gene clusters are poorly understood. In this regard most research has been oriented to infer evolutionary scenarios to explain the sparse phylogenetic 
distribution of some secondary metabolism gene clusters. In many such cases, horizontal gene transfer (HGT) of the entire cluster is suggested as the most plausible scenario ${ }^{19-22}$. One study ${ }^{11}$ explored the evolution of individual genes within metabolic clusters, and found that a minority showed signs of HGT, while most genes had evolved through duplication. However, so far no systematic study has explored how HGT and alternative processes shape gene clusters as a whole. Scenarios that can explain a patchy phylogenetic distribution include HGT, vertical inheritance combined with differential gene loss, as well as independent formation of the same cluster in different lineages. This last scenario of convergent evolution has been proposed to explain the parallel evolution of the galactose utilization (GAL) cluster in yeasts of the Saccharomycotina and Cryptococcus clades $^{23}$, but it is as yet unknown how widespread this process is. Finally, we know very little of the extent of gene clustering beyond secondary metabolism, and whether the functional and evolutionary patterns of gene clustering vary across lineages.

To assess the impact of clustering beyond secondary metabolism, and obtain a broader understanding of evolutionary mechanisms underpinning eukaryotic gene clustering, we performed a comprehensive comparative analysis of 341 fungal genomes. For this, we used an evolutionary-based algorithm, EvolClust, that infers gene clusters independently of the function of the clustered genes ${ }^{24}$. Our analyses defined a catalogue of fungal gene clusters comprising 118,699 clusters, which can be grouped into 12,120 families with varying distributions. Using phylogenetics, we determined the most plausible evolutionary scenario that could explain the phylogenetic distribution of each cluster. Altogether, our study provides new insights on the evolution of eukaryotic gene clusters, and sets the path for the discovery of novel functions associated to gene clusters. 


\section{Results}

\section{Widespread gene clustering in fungi}

We retrieved 341 fungal genomes (Supplementary Table 1), representing a broad but unbalanced taxonomic sampling (Supplementary Figure 1). We used EvolClust ${ }^{24}$ to predict evolutionarily conserved gene clusters and group them into families (see Materials and Methods, and Supplementary Discussion for considerations on factors affecting this algorithm).

This algorithm detected 12,120 gene cluster families (CFs), which comprised 118,699 individual clusters -2 to 148 clusters per CF-, and 1,100,163 genes (see Supplementary Table 2). Cluster families can also be browsed through evolclustDB (http://evolclustdb.org). This represents $\sim 32 \%$ of the gene space in our 341 genomes. Even considering only proteins found in at least half of the clusters in a family, the number of clustered genes remains high at 949,973 (27\%). This number is higher than estimates made considering only enzimatic genes ${ }^{11}$, but is similar to other estimates on a smaller dataset ${ }^{25}$, and to predictions by the alternative method Gecko $3^{26}$ in the same dataset (see Supplementary Discussion). Note that predictions may be influenced by several factors (see Supplementary Discussion).

For comparative purposes, we defined a restrictive set of CFs comprising only 1,704 CFs present in at least two different taxonomic classes hereafter widespreadCF, see Methods), which is expected to comprise the most reliable CFs, but will miss true clusters with restricted taxonomic distribution. In this restrictive dataset the fraction of clustered genes decreased to 7.4\%. Only four species in our dataset did not present any cluster (Batrachochytrium dendrobatidis, Rozella allomycis, Wallemia ichthyophaga and Wallemia sebi), which we attribute to the current poor sampling in those lineages. On the other end of the spectrum 
Fusarium oxysporum presented the largest number of clusters (680).

To assess how clustering varies across lineages, we assigned CFs to taxonomic levels (see Methods), and assessed distribution above the class level. This distribution is uneven, with most clusters mapping to the Pezizomycotina subphylum $(7,200,60 \%)$. Eurotiomycetes (Pezizomycotina), is the class with more CFs (2,143, 21\%), followed by Agaricomycetes (Agaricomycotina) $(2,104,20 \%)$. To account for differences in genome size we calculated the fraction of clustered genes per genome (Figure 1). Saccharomycotina have the largest fraction of clustered genes (median of $50 \%$ ), followed by Pezizomycotina (40\%). Altogether, these results suggest that gene clustering is rampant in fungi, involving a very diverse set of CFs unevenly distributed across clades.

We tested for functional enrichment among clustered genes using Gene Ontology (GO) annotations (see Supplementary Table 3). A significant proportion of enriched terms were related to intracellular protein transport, including vacuolar transport, vesicle-mediated transport, protein import into mitochondrial matrix. Other prominent categories were cellular protein catabolism and sugar metabolism. Similar results were obtained in the widespreadCF dataset (see Supplementary Table 4). We also tested whether gene clusters contained transcription factors (TFs) that could be implicated in their regulation. Roughly one third of genes annotated as putative TFs were encoded within CFs (18,702 - 35\%), which is significantly more than expected by chance $(17,332,32 \%$, p-value $<2.2 \mathrm{e}-16)$. However, most clusters contained no TFs $(9167,76 \%)$, which suggests most gene clusters -if co-regulated- are regulated distally. This trend was confirmed in the widespreadCF dataset, where only $11 \%$ of the clusters encoded TFs. 
We focused on CFs where TFs were commonly present (Supplementary Table 5) and tested for functional enrichment (see Supplementary Table 6). Unsurprisingly the most prominent enriched term was related to regulation of transcription. Other enriched categories were mitochondrial inheritance, chromosomal segregation and glucose metabolism.

\section{Evolution of gene clusters.}

As mentioned, gene duplication has been proposed as the main evolutionary mechanisms among genes in clusters ${ }^{11}$. However, this is based on analyses restricted to metabolic clusters, and relates to individual genes, not to entire clusters. We here used phylogenomics to assess evolutionary processes affecting both individual genes and entire clusters, independently of their function. Our approach involved reconstructing a phylogenetic tree for each gene family present in a cluster (see Methods). We found that roughly $70 \%$ of the clustered genes were duplicated at least once in their lineage. This value is lower than what was previously described for metabolic clusters ${ }^{11}(90 \%)$. Additionally, we noticed a limited impact of duplications of entire clusters, affecting only 639 (5\%) of CFs. Unsurprisingly, the three species with the largest amount of duplicated clusters (adding up to roughly half of the total number of duplicated clusters) were the allotetraploid Zygosaccharomyces parabailii ${ }^{27}$ and the two Rhizopus species that underwent a recent whole genome duplication ${ }^{28}$. Yeasts related to Saccharomyces cerevisiae descend from an allotetraploid ${ }^{29}$, however, in this case, time seems to have eroded the presence of duplicted clusters. Thus duplication of entire clusters can occur, although at very low rates unless they are associated with recent polyploidizations.

Another puzzling observation for metabolic gene clusters is that they often have very sparse phylogenetic distributions ${ }^{19-21,30-33}$, which has been attributed to be the consequence of HGT. 
However, it is unclear whether this is the general case. Alternative evolutionary scenarios potentially resulting in sparse distributions include differential loss, as well as convergent formation of gene clusters. To assess this, we evaluated the plausibility of alternative scenarios (vertical evolution, convergent evolution, and HGT) using a newly developed algorithm called EvolClassifier (see Supplementary Figure 2A, Methods, and Supplementary Discussion) that considers i) the phylogenetic distribution of the cluster and the enocoded protein families ii) the species phylogeny iii) the gene phylogeny and iv) the internal gene order conservation. Illustrative examples of CFs whose main mode of evolution has been classified by this algorithm as vertical evolution, HGT, and convergent evolution are shown in Figures 2, 3 and 4 , respectively.

To assess the robustness of the reconstructed scenarios, EvolClassifier repeats the analysis over a range of thresholds, and reports what percentage of trials support each alternative scenario. This can be used as a confidence threshold, and we created two datasets using the $50 \%$ (dataset50, 10,786 CFs) or 75\% (dataset75, 6,875 CFs) threshold (see Supplementary Table 7). Of note, assignment to different evolutionary scenarios was not significantly affected by levels of assembly fragmentation (see Supplementary Discussion). As expected, the most frequent assigned mechanism was vertical evolution (69.5\% for dataset50 and $76.8 \%$ for dataset75). However, a sizable fraction of CFs are inferred to have evolved through convergent evolution (26.2\% for dataset50 and $20.5 \%$ for dataset75). A comparatively small number of CFs (468, $4.3 \%$ for dataset50 and $182,2.7 \%$ for dataset 75 ) is inferred to have evolved mainly through HGT (see Figure 5a and Supplementary Figure 3). The picture is radically different for the widespreadCF group (see Figure 5a and Supplementary Figure 3). In dataset50 only $32.1 \%$ of the assigned clusters evolved through vertical evolution whereas the primary mechanism is convergent evolution (53.6\%). The number of HGT events is also higher (14.3\%). Results for 
dataset 75 followed the same trend.

We compared the average physical size of the different families and found that clusters that have been horizontally transferred or that evolved through convergent evolution were significantly smaller than those evolving vertically (Wilcoxon test, p-value $<2.2 \mathrm{e}-16$ ). We also measured branch lengths (see methods), and found that fast evolving genes can be more often found in clusters that have evolved through HGT (Wilcoxon test, p-value $<2.2 \mathrm{e}-16$ ), which may reflect the accumulation of changes required to adapt to the recipient genome.

\section{Different impact of gene cluster evolutionary processes across fungal lineages}

We mapped CFs onto the species phylogeny as explained above, this time binning by evolutionary mechanism. As seen in Figure 5b and Supplementary Figure 3, clusters mapping at different taxonomic groups have a tendency to maintain gene order conservation through different evolutionary mechanisms. Clusters in Microsporidia, Mucoromycotina, Pucciniomycotina, Ustilaginomycotina and Taphrinomycotina have evolved preferentially through vertical evolution (range between $100 \%$ and $89.9 \%$ in dataset50 and between $95.0 \%$ and $84.0 \%$ in dataset75). However, this might be partly due to their smaller sampling representation, which hampers the detection of more complex scenarios. Pezizomycotina, Saccharomycotina and Agaricomycotina contain a majority of CFs that evolved vertically, yet the percentage of families that evolved through convergent evolution is much higher (range between $14.7 \%$ and $35.0 \%$ in dataset50 and $7.5 \%-19.7$ in dataset 75$)$. Curiously Agaricomycotina have the largest amount of CFs that evolved through convergent evolution. There does not seem to be any sampling bias that could explain this effect. Agaricomycotina contains a large number of species, but less than Pezizomycotina and taxonomic diversity is 
also larger in Pezizomycotina.

Finally, most clusters that evolved through HGT are found within Pezizomycotina (387 out of the 468 detected, $82.7 \%$ in dataset50 ). Other groups that contain cluster families that evolved through HGT are Microsporidia (1), Ustilaginomycotina (5), Saccharomycotina (25), and Agaricomycotina (48). This trend is maintained in dataset75. This imbalance in the number of HGT events is in line with previous studies of individual genes ${ }^{34}$. Thus Pezizomycotina seem to be particularly prone to HGT, including transference of entire gene clusters.

\section{Functional characteristics of differently evolving cluster families.}

All CFs were associated to at least one COG function (see Supplementary Table 8). We assessed whether different evolutionary mechanisms preferentially affected certain functions. Twelve COG categories were enriched among clusters evolving mostly through vertical evolution in dataset50 (see Supplementary Table 9), with most of them related to information storage and processing (100\% of the terms in the category are enriched) and to cellular processes and signaling (50\%). Two additional terms related to metabolism were enriched: E (Amino acid metabolism and transport) and F (Nucleotide transport and metabolism). In dataset75 fewer terms were enriched (9), of which none belonged to metabolism. On the other hand, in both datasets CFs that evolved through HGT were only enriched in secondary metabolism biosynthesis, transport and catabolism (Q). CFs that evolved through convergent evolution were not enriched in any particular functional category.

We also performed a GO term enrichment analyses, comparing the individual genes within CFs that evolved through each of the three mechanisms, as compared to the whole set of clustered 
genes. For dataset50, families that evolved through vertical evolution were only enriched in 10 terms, related to protein phosphorylation, DNA replication and translation (see Supplementary Table 10). Gene families that evolved through HGT were enriched in 58 terms (see Supplementary Table 11), all directly related to metabolic processes. Finally, families that evolved through convergent evolution were enriched in 122 GO terms (see Supplementary Table 12), many of which related to metabolic processes but also to operational terms such as ribosome biogenesis or kinetochore assembly. Enrichments for dataset75 differed slightly with an increase in enriched GO terms in families that evolved vertically. Terms were mainly related to protein phosphorylation, regulation and chromatin remodeling. Terms for the other categories were similar (see Supplementary Table 13, 14 and 15).

We also checked whether clusters that evolved through HGT were more likely to encode their own TF as reported for gamma-proteobacteria ${ }^{35}$. Only 52 of the clusters involved in HGT in dataset50 contained a TF (10.9\%). A similar percentage is observed for clusters that went through convergent evolution (11.9\%), while this is somewhat higher for CFs that evolved vertically $(16.8 \%)$. Similar results were obtained for dataset 75 . We relaxed our requirements by including clusters were TFs could be found up to five genes away of the predicted clusters, which increased the number of clusters with TFs to $92(19.6 \%)$ in the case of HGT, $525(18.6 \%)$ for CE and 1851 (24.7\%) for VE. So there does not seem to be any particular preference for clusters with a TF to evolve through a given mechanism. This observation also implies that clusters that have been transferred could be regulated by an existing TF in the recipient genome.

\section{Fine-grained evolutionary analysis}

One drawback of the used approach is that it assigns one main evolutionary mode to each 
cluster family, whereeas in reality it may have evolved by a combination of evolutionary processes. For instance, families classified as HGT may show long periods in which the cluster was simply vertically transmitted. In other cases, a cluster that may have been formed twice independently can still have been transferred to yet another lineage. This is the case of the GAL cluster which evolved the novo in Candida albicans and in Cryptococcus but was also transferred from Candida to Schizosaccharomyces ${ }^{23}$. We developed a more fine-grained approach which defines an evolutionary mode per each pairwise comparison of the species having the cluster (see Methods), and used it to re-assess all CFs that had not evolved vertically (4585 families for dataset50 and 6,800 families for dataset75, see Supplementary Table 16). If we add these results to the ones obtained previously we observe that, expectedly, most cluster families (85.5\% in dataset50) show vertical evolution in at least one pairwise comparison. The number of cluster families that include convergent evolution as one of the mechanisms increases only slightly from $23.4 \%$ to $27.7 \%$, whereas HGT nearly doubles (from $3.9 \%$ to 6.9\%). A similar trend is observed in dataset75. This shows that, while HGT may not be the main mechanism through which CFs evolve, in some cases it is still involved during part of the evolution. These results also confirm an important role to convergent evolution.

We assessed these patterns in the widespreadCF dataset, which showed important differences. In this dataset, the amount of clusters that have, at least partially, evolved through vertical evolution decreases to $73.5 \%$ whereas those that have some clusters that evolved through convergent evolution increased to 57.2\%. The fraction of CFs that included HGT also increases from $6.9 \%$ to $22.1 \%$ in this subset. Same trends are observed in dataset75. Thus, while vertical evolution remains the main evolutionary mode, convergent evolution and HGT are likely to explain a sizable fraction of clusters that are spread across taxonomic classes. 


\section{Horizontal transfer of clustered genes preferentially affects secondary metabolism}

We matched our inferred CFs with a list of 132 known secondary metabolite gene clusters retrieved from the literature (see Supplementary Table 17), and identified 40 of them in our dataset (see Supplementary Table 18). In addition we used the presence of signal proteins (NPRS, PKS and DMAT proteins) predicted by $\mathrm{SMURF}^{36}$ and $\mathrm{SMIPS}^{37}$ as indicators of a secondary metabolism cluster (see Supplementary Table 19).

The combination of both methods results in a total of 391 secondary metabolism clusters, which represents a very small fraction of the total number of inferred clusters $(3.2 \%, 9 \%$ in widespreadCF). Most secondary metabolism clusters map to Pezizomycotina (313-80\%), followed by Saccharomycotina and the Agaricomycotina subphylums with 27 cluster families each $(7 \%)$.

Most secondary metabolism clusters evolved through vertical evolution (39.6\%), which is much lower than in the overall dataset (62.0\%) (see Figure 5a). The difference can be attributed to the comparatively larger amount of CFs that evolved through HGT, as compared to the overall set of clusters $(25.8 \%$ vs $3.9 \%)$. When considering fine-grained scenarios, we find signs of vertical evolution in most families (287 - 73.4\%) and of HGT in $138(35.35 \%)$ of the families. Intriguingly, the evolutionary patterns found in the 40 known cluster families are the most deviating with two thirds showing signs of HGT. 28 out of these 40 families evolved through at least two of the evolutionary mechanisms considered and 14 used all three of them. These values are far above what has been shown in any other tested dataset and can partially explain the preponderance of HGT described in secondary metabolism clusters over the last $\operatorname{decade}^{22}$. 


\section{Conclusions}

Our results suggest a high level of gene clustering, with roughly one third of the fungal gene space belonging to 12,120 evolutionarily conserved CFs. As our algorithm uses thresholds that define how unexpected an observed shared neighborhood is, with any threshold defining a trade-off between false positives and false negatives. We based our threshold choice based on the ability to detect known metabolic clusters, among other considerations (see Supplementary discussion), and assessed the robustness of the observed trends by using various levels of stringency. All in all we consider our predicted catalogue of shared gene clusters a useful set to study functional and evolutionary trends that underlie gene neighborhood. We expect this dataset to comprise a significant fraction of clusters encoding potentially co-regulated and functionally related genes, whose roles remain to be determined.

Using our set of predicted shared gene clusters, we could uncover differential trends from the perspective of their functionality, phylogenetic distribution, and evolutionary history. Although all fungal clades present evolutionary conserved gene clusters, they do so differentially, with Pezizomycotina and Agaricomycotina showing the genomes with the highest levels of clustering. It is unclear whether this might be related to underlying differences in rates of genome re-organizations. In this regard some filamentous fungi have display a peculiar for of gene order evolution, called mesosynteny ${ }^{38}$, in which genes do not conserve exact gene order but tend to remain in the same chromosome.

We found that gene clustering differentially impacts functional classes so that most enriched terms belong to categories related to metabolism. Most previous studies of gene clustering in 
fungi concern secondary metabolism gene clusters, which are also included in this dataset. They represent a very small fraction (3.2\%) of the evolutionary conserved gene clusters. An important observation is that roughly two thirds of the gene clusters do not encode a transcription factor, which suggestsregulation in trans. This is also true for clusters that have been transferred horizontally, which indicates that following the transfer, other resident transcription factors have been co-opted for their regulation.

Although gene duplication has been found to be common among genes in metabolic gene clusters $^{11}$, our results point to a more limited overall impact. A reduced number of clusters $(5.3 \%)$ are duplicated as a whole, and this is often linked to recent whole genome duplications. We reconstructed the most likely evolutionary scenarios for each of the predicted CFs and found that vertical evolution followed by differential loss was the main evolutionary process. However, our results point to a very relevant role of convergent evolution, which would involve the parallel formation of the same gene cluster in different lineages. The role of HGT was overall sparse, but it was overrepresented in secondary metabolism.

\section{Methods}

\section{Data collection and prediction of gene clusters}

We downloaded all annotated fungal genomes available at NCBI (Last accessed April, 2017). When genomes from multiple strains of the same species were available, we only retained the one for the reference strain, or, -when no reference strain was available or had poor quality- the assembly with the lowest amount of contigs. We discarded fragmented genome assemblies with more than 2,000 scaffolds. The final dataset comprised a total of 341 fungal genomes 
(Supplementary Table 1). Gene annotations were then re-coded so that they provided information on the relative position in the assembly of each annotated protein-coding gene (SpeciesName_ContigNum_ProteinNum). Where SpeciesName is a five letter code unique for each species; ContigNum refers to the contig number in the source assembly; and ProteinNum indicates the relative order of the corresponding protein-coding gene in the genome, where consecutive proteins have correlative numbers. The final proteome database contained $3,421,149$ proteins, which were clustered into families using a all-vs-all blastp ${ }^{39}$, followed by clustering with MCL 14-137 $7^{40}$, using an inflation parameter of 2.0. Evolutionarily conserved gene clusters and cluster families were inferred using EvolClust V1.024. EvolClust was run using a minimum cluster size of 5 genes and a maximum cluster size of 35 genes. Up to three non-homologous genes could be found in between any pair of homologous proteins. The thresholds to distinguish between clusters and gene order conservation were calculated using the 2 standard deviations option implemented in EvolClust. The number of predicted clusters as well as the cluster sizes will be dependant on the thresholds used in this analysis.

\section{Taxonomic mapping of gene clusters}

Predicted clusters were related to the NCBI taxonomy tree by associating each cluster family to the common ancestor of the species in which it was present. For instance, if a cluster is found in Aspergillus clavatus and in Penicillium expansum, this cluster will be mapped as present in all the lineages and taxonomic levels from the common ancestor of these species towards the root (i.e. Aspergillaceae, Eurotiales, Eurotiomycetes, Pezizomycotina, Ascomycota and Fungi).

\section{Gene tree reconstruction.}

A phylogenetic tree was reconstructed for every protein family found in the set of predicted gene clusters. A total of 60,955 trees were reconstructed. For large protein families $(>300$ 
members) a fast approach was used in which alignments were reconstructed with MAFFT $(\mathrm{v} 6.861 \mathrm{~b})^{41}$ and gene trees with fasttree $\mathrm{v} 2 \cdot 1.10^{42} .2995$ families (5\%) were analysed using this approach. For smaller families ( $<300$ members) a more accurate method was used based on the PhylomeDB pipeline ${ }^{43}$. In brief, six multiple sequence alignments are performed using three different programs (MUSCLE v3.8 ${ }^{44}$, MAFFT v6.861 $\mathrm{b}^{41}$ and KALIGN 2.04 ${ }^{45}$ ) in forward and reverse direction ${ }^{46}$. A consensus alignment is then reconstructed using M-coffee ${ }^{47}$ and then trimmed with trimAl v1.4 (-cs 0.16667 and - gt 0.1$)^{48}$. The resulting alignment was then used to reconstruct neighbour joining trees as implemented in $\mathrm{BIONJ}^{49}$ and the likelihood of seven different evolutionary models was assessed. Finally, the model with the best likelihood according to the AIC criterion ${ }^{50}$ was used to reconstruct a maximum likelihood tree using phyML v3.0 $0^{51}$.

Branch length distances were calculated by measuring the branch length of each protein encoded in a species across all different gene trees. The average branch length per species was calculated and then individual branch lengths were corrected with this measure to see whether they evolved at a different rate than the average.

\section{Species tree reconstruction.}

In order to reconstruct the species tree we used the treeKO algorithm ${ }^{52}$ as implemented in ETE $\mathrm{v} 3.0^{53}$ to obtain subsets of widespread orthologous proteins from gene trees. This algorithm decomposes a tree with duplications and speciation nodes into subtrees that only contain orthologous proteins. Of the multiple pruned sub-trees derived from a gene tree, the one comprising the largest number of species was chosen. Sub-trees containing less than 200 species were discarded. If multiple sub-trees complied with these parameters, one was chosen at random. The orthologous sequences contained within each of the selected sub-trees were 
then aligned using MAFFT (v6.861b) and concatenated into a single alignment. The final, concatenated dataset comprised orthologous sequences from 442 protein families, and a total of 194,225 amino acid positions. Fasttree v2.1.10 ${ }^{42}$ with default parameters was used to reconstruct the species tree (Supplementary Figure 1). The resulting species tree is highly congruent with the known taxonomic classification of fungi.

\section{Evolutionary assessment - EvolClassifier}

In order to assess the most plausible evolutionary scenario accounting for the observed phylogenetic distribution of each gene cluster family (CF) we developed EvolClassifier. This algorithm jointly considers: i) the species tree; ii) the phylogenetic distribution of a cluster family and, iii) the phylogenies of each gene family that is part of the cluster. For each CF it considers all gene families that are present in at least two clusters of the family. For each such gene family (GF) it assesses (see Supplementary Figure 4 for details) the following parameters:

1- Monophyly in the species tree: Takes a binary value (Yes/No) depending on whether the species in the $\mathrm{CF}$ are monophyletic in the species tree shown in Supplementary Figure 1 (see Supplementary Figure 4b).

2- If the species in CF are not monophyletic, the number of implied losses necessary to explain the scenario, assuming the cluster was gained at the common ancestor of the species in CF and that vertical inheritance is the only evolutionary mechanism. The minimum number of losses needed to explain the distribution of CF is calculated based on parsimony (see Supplementary Figure 4c).

3- Monophyly in the gene tree: Takes a binary value (Yes/No) depending on whether the GF 
sequences belonging to CF are monophyletic in the gene tree (see Supplementary Figure 4d). 4.- Given the subtree that contains all GF genes present in $\mathrm{CF}$, the number of additional GF genes found in the subtree that are not present in CF (see Supplementary Figure 4e).

EvolClassifier (see Supplementary Figure 2a) will assign an evolutionary mode to the gene family, choosing among: i) vertical evolution; ii) convergent evolution; or iii) HGT based on two thresholds: Threshold P and Threshold L.4 Threshold L is related to the number of inferred losses detected when the species in the species tree are not monophyletic (see Supplementary Figure 4c), while threshold $\mathrm{P}$ is related to the number of additional genes detected (see Supplementary Figure 4e). Thresholds should be related to the size of each cluster, therefore EvolClassifier repeats the same analysis using an array of variable factors that allows it to test different threshold values. To define threshold $\mathrm{P}$ the number of additional genes (see point 4 above) is multiplied by a factor that ranges from 2.0 to 5.0 by increments of 0.25 . Threshold $\mathrm{L}$ is defined by multiplying the number of predicted losses (see point 2 above) by a factor that ranges from 1.0 to 2.0 in increments of 0.25 . A total of 48 analyses are executed for each gene family. A table is provided indicating the percentage of analyses that supported each of the three scenarios for all the gene families in a CF. In order to perform posterior analyses a main evolutionary mechanism is assigned to each CF based on the percentage of analyses that support each mechanism. In dataset50, more than $50 \%$ of the analyses need to support a method for it to be assigned, else the CF will be annotated as undecided. Similarly, for dataset75 the decision threshold is set at $75 \%$ of the analyses. There is one exception. When the internal gene order of the $\mathrm{CF}$ is conserved and the main mechanism assigned is convergent evolution, this will be changed to undecided. Cluster gene order is calculated when possible with $\mathrm{MGR}^{54}$. MGR needs genes to be present in all species without duplications. (see Supplementary Figure 
$2 b)$.

EvolClassifier has a second, more thorough evolutionary analysis for CFs that did not evolve through vertical evolution. This detailed evolutionary analysis is performed by splitting CFs into all pairwise combinations of clusters. For each pair of clusters EvolClassifier follows the same approach as described above to determine their evolutionary mechanism as if they were the only two clusters present. The only adjustment is that when calculating the number of additional genes found in the gene tree (parameter 4 described above) all clusters in the CF are considered in order to not over-estimate the number of genes not in the cluster. Once all pairwise assessments are done, EvolClassifier performs a UPGMA clustering using the python module hclust to join those clusters that evolved through vertical evolution and then it repeats the analysis between all cluster groups that have not evolved through vertical evolution. This is repeated until no vertical evolution events can be predicted between the remaining groups of clusters. EvolClassifier is distributed with EvolClust ${ }^{24}$ at github (https://github.com/Gabaldonlab/EvolClust/tree/master/additional_scripts/).

\section{Functional characterization of gene clusters and enrichment analysis.}

We performed a functional analysis for each protein in our database using interproscan (version 5.23-62.0 $)^{55}$. GO terms were taken from interproscan and COG functional categories were inferred using eggnog-mapper v0.12.7 $7^{56}$. Transcription factors were identified by searching for the Pfam domains ${ }^{57}$ associated to transcription factors described in DBD Transcription factor prediction database ${ }^{58}$ using HMMER v3.1b2 ${ }^{59}$. Cluster families were annotated as having a TF when more than half of its cluster contained a TF. In a broader analysis clusters were also annotated as having a TF if one was found 5 genes upstream or downstream from a 
characterized cluster. Functional enrichment analyses were performed using an in-house adaptation of FatiGO ${ }^{60}$. Enriched terms were accepted at a corrected p-value $<0.001$ (two sided). Enriched GO terms were afterwards filtered with ReviGO ${ }^{61}$.

Cluster families that likely produced secondary metabolites were identified as follows: First $\mathrm{SMURF}^{36}$ and $\mathrm{SMIPS}^{37}$ were used to detect signal proteins in all our proteomes. Then cluster families that contained these signal proteins were tagged as candidates. We only considered cluster families where the signal protein was present in at least $50 \%$ of the clusters within the family, unless the cluster family only contained 3 or 2 clusters, in which case the signal protein was required to be in two of the clusters.

Known clusters were identified using a list of 132 known clusters (updated from ${ }^{62}$ (see Supplementary Table 2)) and performing blastP searches of the known cluster proteins against the different proteomes. Then the list of clusters was matched to the cluster families.

\section{Data availability.}

All proteomes were downloaded from NCBI (April, 2017). The full list of BioProjects can be found in Supplementary Table 1. EvolClust can be downloaded from the github repository: (https://github.com/Gabaldonlab/EvolClust/) and Evolclassifier is found within the folder called additional scripts within this repository. Any further data is available from the corresponding author upon request.

\section{Corresponding author}

Correspondence to Toni Gabaldón.

\section{Acknowledgments}


TG group acknowledges support from the Spanish Ministry of Economy, Industry, and Competitiveness (MEIC) for the EMBL partnership, and grants 'Centro de Excelencia Severo Ochoa 2013-2017' SEV2012-0208, and BFU2015-67107 cofounded by European Regional Development Fund (ERDF); from the CERCA Programme / Generalitat de Catalunya; from the Catalan Research Agency (AGAUR) SGR857, and grant from the European Union's Horizon 2020 research and innovation programme under the grant agreement ERC-2016-724173 the Marie Sklodowska-Curie grant agreement No H2020MSCA-ITN-2014-642095.

\section{Author contributions}

TG and MMH designed the study, MMH gathered the data and performed the cluster prediction and phylogenomics analysis. TG and MMH analysed the results and wrote the manuscript. TG supervised the study.

\section{Competing interests}

The authors declare no competing interests

\section{References}

1. Osbourn, A. E. \& Field, B. Operons. Cell. Mol. Life Sci. 66, 3755-3775 (2009).

2. Hurst, L. D., Pál, C. \& Lercher, M. J. The evolutionary dynamics of eukaryotic gene order. Nat. Rev. Genet. 5, 299-310 (2004).

3. Dávila López, M., Martínez Guerra, J. J. \& Samuelsson, T. Analysis of gene order conservation in eukaryotes identifies transcriptionally and functionally linked genes. PLoS One 5, e10654 (2010).

4. Cohen, B. A., Mitra, R. D., Hughes, J. D. \& Church, G. M. A computational analysis of 
whole-genome expression data reveals chromosomal domains of gene expression. Nat. Genet. 26, 183-186 (2000).

5. Wang, G.-Z., Chen, W.-H. \& Lercher, M. J. Coexpression of linked gene pairs persists long after their separation. Genome Biol. Evol. 3, 565-570 (2011).

6. Boutanaev, A. M., Kalmykova, A. I., Shevelyov, Y. Y. \& Nurminsky, D. I. Large clusters of co-expressed genes in the Drosophila genome. Nature 420, 666-669 (2002).

7. Reimegård, J. et al. Genome-wide identification of physically clustered genes suggests chromatin-level co-regulation in male reproductive development in Arabidopsis thaliana. Nucleic Acids Res. 45, 3253-3265 (2017).

8. Poyatos, J. F. \& Hurst, L. D. Is optimal gene order impossible? Trends Genet. 22, 420423 (2006).

9. Teichmann, S. A. \& Veitia, R. A. Genes encoding subunits of stable complexes are clustered on the yeast chromosomes: an interpretation from a dosage balance perspective. Genetics 167, 2121-2125 (2004).

10. Lee, J. M. \& Sonnhammer, E. L. L. Genomic gene clustering analysis of pathways in eukaryotes. Genome Res. 13, 875-882 (2003).

11. Wisecaver, J. H., Slot, J. C. \& Rokas, A. The evolution of fungal metabolic pathways. PLoS Genet. 10, e1004816 (2014).

12. Hull, E. P., Green, P. M., Arst, H. N., Jr \& Scazzocchio, C. Cloning and physical characterization of the L-proline catabolism gene cluster of Aspergillus nidulans. Mol. Microbiol. 3, 553-559 (1989).

13. Keller, N. P. \& Hohn, T. M. Metabolic pathway gene clusters in filamentous fungi. Fungal Genet. Biol. 21, 17-29 (1997).

14. Johnston, M. A model fungal gene regulatory mechanism: the GAL genes of 
Saccharomyces cerevisiae. Microbiol. Rev. 51, 458-476 (1987).

15. Wong, S. \& Wolfe, K. H. Birth of a metabolic gene cluster in yeast by adaptive gene relocation. Nat. Genet. 37, 777-782 (2005).

16. Hoffmeister, D. \& Keller, N. P. Natural products of filamentous fungi: enzymes, genes, and their regulation. Nat. Prod. Rep. 24, 393-416 (2007).

17. Keller, N. P. Translating biosynthetic gene clusters into fungal armor and weaponry. Nat. Chem. Biol. 11, 671-677 (2015).

18. Takos, A. M. \& Rook, F. Why biosynthetic genes for chemical defense compounds cluster. Trends Plant Sci. 17, 383-388 (2012).

19. Slot, J. C. \& Rokas, A. Horizontal transfer of a large and highly toxic secondary metabolic gene cluster between fungi. Curr. Biol. 21, 134-139 (2011).

20. Campbell, M. A., Rokas, A. \& Slot, J. C. Horizontal transfer and death of a fungal secondary metabolic gene cluster. Genome Biol. Evol. 4, 289-293 (2012).

21. Khaldi, N. \& Wolfe, K. H. Evolutionary Origins of the Fumonisin Secondary Metabolite Gene Cluster in Fusarium verticillioides and Aspergillus niger. Int. J. Evol. Biol. 2011, $423821(2011)$.

22. Wisecaver, J. H. \& Rokas, A. Fungal metabolic gene clusters-caravans traveling across genomes and environments. Front. Microbiol. 6, 161 (2015).

23. Slot, J. C. \& Rokas, A. Multiple GAL pathway gene clusters evolved independently and by different mechanisms in fungi. Proc. Natl. Acad. Sci. U. S. A. 107, 10136-10141 (2010).

24. Marcet-Houben, M. \& Gabaldón, T. Evolclust: automated inference of evolutionary conserved gene clusters in eukaryotes. bioRxiv 698621 (2019).

25. Ohm, R. A. et al. Diverse lifestyles and strategies of plant pathogenesis encoded in the 
genomes of eighteen Dothideomycetes fungi. PLoS Pathog. 8, e1003037 (2012).

26. Winter, S. et al. Finding approximate gene clusters with Gecko 3. Nucleic Acids Res. 44, 9600-9610 (2016).

27. Ortiz-Merino, R. A. et al. Evolutionary restoration of fertility in an interspecies hybrid yeast, by whole-genome duplication after a failed mating-type switch. PLoS Biol. 15, e2002128 (2017).

28. Ma, L.-J. et al. Genomic analysis of the basal lineage fungus Rhizopus oryzae reveals a whole-genome duplication. PLoS Genet. 5, e1000549 (2009).

29. Marcet-Houben, M. \& Gabaldón, T. Beyond the Whole-Genome Duplication: Phylogenetic Evidence for an Ancient Interspecies Hybridization in the Baker's Yeast Lineage. PLoS Biol. 13, e1002220 (2015).

30. Reynolds, H. et al. Differential retention of gene functions in a secondary metabolite cluster. Mol. Biol. Evol. (2017). doi:10.1093/molbev/msx145

31. Khaldi, N., Collemare, J., Lebrun, M.-H. \& Wolfe, K. H. Evidence for horizontal transfer of a secondary metabolite gene cluster between fungi. Genome Biol. 9, R18 (2008).

32. Druzhinina, I. S., Kubicek, E. M. \& Kubicek, C. P. Several steps of lateral gene transfer followed by events of 'birth-and-death' evolution shaped a fungal sorbicillinoid biosynthetic gene cluster. BMC Evol. Biol. 16, 269 (2016).

33. Marcet-Houben, M. \& Gabaldón, T. Horizontal acquisition of toxic alkaloid synthesis in a clade of plant associated fungi. Fungal Genet. Biol. 86, 71-80 (2016).

34. Marcet-Houben, M. \& Gabaldón, T. Acquisition of prokaryotic genes by fungal genomes. Trends Genet. 26, 5-8 (2010).

35. Price, M. N., Dehal, P. S. \& Arkin, A. P. Horizontal gene transfer and the evolution of 
transcriptional regulation in Escherichia coli. Genome Biol. 9, R4 (2008).

36. Khaldi, N. et al. SMURF: Genomic mapping of fungal secondary metabolite clusters. Fungal Genet. Biol. 47, 736-741 (2010).

37. Wolf, T., Shelest, V., Nath, N. \& Shelest, E. CASSIS and SMIPS: promoter-based prediction of secondary metabolite gene clusters in eukaryotic genomes. Bioinformatics 32, 1138-1143 (2016).

38. Hane, J. K. et al. A novel mode of chromosomal evolution peculiar to filamentous Ascomycete fungi. Genome Biol. 12, R45 (2011).

39. Altschul, S. F., Gish, W., Miller, W., Myers, E. W. \& Lipman, D. J. Basic local alignment search tool. J. Mol. Biol. 215, 403-410 (1990).

40. Enright, A. J., Van Dongen, S. \& Ouzounis, C. A. An efficient algorithm for large-scale detection of protein families. Nucleic Acids Res. 30, 1575-1584 (2002).

41. Katoh, K., Kuma, K., Toh, H. \& Miyata, T. MAFFT version 5: improvement in accuracy of multiple sequence alignment. Nucleic Acids Res. 33, 511-518 (2005).

42. Price, M. N., Dehal, P. S. \& Arkin, A. P. FastTree 2--approximately maximum-likelihood trees for large alignments. PLoS One 5, e9490 (2010).

43. Huerta-Cepas, J. et al. PhylomeDB v3.0: an expanding repository of genome-wide collections of trees, alignments and phylogeny-based orthology and paralogy predictions. Nucleic Acids Res. 39, D556-60 (2011).

44. Edgar, R. C. MUSCLE: a multiple sequence alignment method with reduced time and space complexity. BMC Bioinformatics 5, 113 (2004).

45. Lassmann, T. \& Sonnhammer, E. L. Kalign--an accurate and fast multiple sequence alignment algorithm. BMC Bioinformatics 6, 298 (2005).

46. Landan, G. \& Graur, D. Heads or tails: a simple reliability check for multiple sequence 
alignments. Mol. Biol. Evol. 24, 1380-1383 (2007).

47. Wallace, I. M., O’Sullivan, O., Higgins, D. G. \& Notredame, C. M-Coffee: combining multiple sequence alignment methods with T-Coffee. Nucleic Acids Res. 34, 1692-1699 (2006).

48. Capella-Gutierrez, S., Silla-Martinez, J. M. \& Gabaldon, T. trimAl: a tool for automated alignment trimming in large-scale phylogenetic analyses. Bioinformatics 25, 1972-1973 (2009).

49. Gascuel, O. BIONJ: an improved version of the NJ algorithm based on a simple model of sequence data. Mol. Biol. Evol. 14, 685-695 (1997).

50. Akaike, H. Information theory and extension of the maximum likelihood principle. in Proceedings of the 2nd international symposium on information theory 267-281 (1973).

51. Guindon, S. et al. New algorithms and methods to estimate maximum-likelihood phylogenies: assessing the performance of PhyML 3.0. Syst. Biol. 59, 307-321 (2010).

52. Marcet-Houben, M. \& Gabaldon, T. TreeKO: a duplication-aware algorithm for the comparison of phylogenetic trees. Nucleic Acids Res. 39, e66 (2011).

53. Huerta-Cepas, J., Serra, F. \& Bork, P. ETE 3: Reconstruction, Analysis, and Visualization of Phylogenomic Data. Mol. Biol. Evol. 33, 1635-1638 (2016).

54. Bourque, G. \& Pevzner, P. A. Genome-scale evolution: reconstructing gene orders in the ancestral species. Genome Res. 12, 26-36 (2002).

55. Jones, P. et al. InterProScan 5: genome-scale protein function classification. Bioinformatics 30, 1236-1240 (2014).

56. Huerta-Cepas, J. et al. Fast genome-wide functional annotation through orthology assignment by eggNOG-mapper. Mol. Biol. Evol. (2017).

57. Finn, R. D., Bateman, A., Clements, J. \& Coggill, P. Pfam: the protein families database. 
Nucleic acids (2013).

58. Wilson, D., Charoensawan, V., Kummerfeld, S. K. \& Teichmann, S. A. DBD-taxonomically broad transcription factor predictions: new content and functionality. Nucleic Acids Res. 36, D88-92 (2008).

59. Eddy, S. R. Accelerated Profile HMM Searches. PLoS Comput. Biol. 7, e1002195 (2011).

60. Al-Shahrour, F., Díaz-Uriarte, R. \& Dopazo, J. FatiGO: a web tool for finding significant associations of Gene Ontology terms with groups of genes. Bioinformatics 20, 578-580 (2004).

61. Supek, F., Bošnjak, M., Škunca, N. \& Šmuc, T. REVIGO summarizes and visualizes long lists of gene ontology terms. PLoS One 6, e21800 (2011).

62. Ballester, A.-R. et al. Genome, Transcriptome, and Functional Analyses of Penicillium expansum Provide New Insights Into Secondary Metabolism and Pathogenicity. Mol. Plant. Microbe. Interact. 28, 232-248 (2015).

\section{Figure Legends:}

Figure 1: Sunburst showing the percentage of clustered protein-coding genes per species. Outer ring indicates individual species. Colour density indicates percentage of proteins in cluster versus total number of proteins in proteome. Internal rings represent taxonomic groups. Colour density is obtained by calculating the median of the fraction of clustered proteins for all the species belonging to the taxonomic category.

Figure 2: Example of gene cluster family classified as vertically evolving. They tend to be 
present in closely related species. A.- Represents the node in the species tree that contains all the species that have the cluster family. On the right is a graphical representation of the cluster family, where each square represents a predicted gene and the colour shows homologous gene families. White squares represent lack of homology within the cluster family. B.- Examples of the gene trees belonging to three of the gene families. Leaf names in blue are those found in the cluster family. The coloured square on top of the tree indicates to which gene in the cluster family the tree belongs to.

Figure 3: Example of gene cluster family classified as HGT. CFs that have undergone a HGT are found sparsely in far related species but the gene trees indicate a close relationship between gene belonging to the CF. A.- Species tree representing the common ancestor of the species that contain the cluster family. Red stars indicate which species contain the family. Scheme in the right represents the gene cluster family as described in Figure 2. B.- Examples of gene trees as shown in Figure 2.

Figure 4: Example of gene cluster family that has undergone convergent evolution. CFs that evolved through convergent evolution are spread out in the gene tree instead of grouping together. a.- Species tree representing the common ancestor of the species that contain the cluster family. Colours in the species tree indicate different taxonomic groups. On both sides of the tree there is a schematic representation of the gene cluster family as described in Figure 2. In this case genes found in the two clusters are coloured in blue and red, one for each species. b.- Examples of gene trees as shown in Figure 2.

Figure 5: A.- Pie charts representing the percentage of clusters that evolved primarily through 
a given evolutionary mechanism in dataset50. Charts are build for all cluster families, the widespreadCF subset, the group of cluster families predicted to encode secondary metabolism gene clusters, and those of the last category that have a known function. B.- Percentage of clusters that follow each of the three considered evolutionary mechanisms in each subphylum for dataset50. Numbers on the right of the graph represent the total number of cluster families that map within each subphylum and the average percentage of proteins in cluster in those species. 


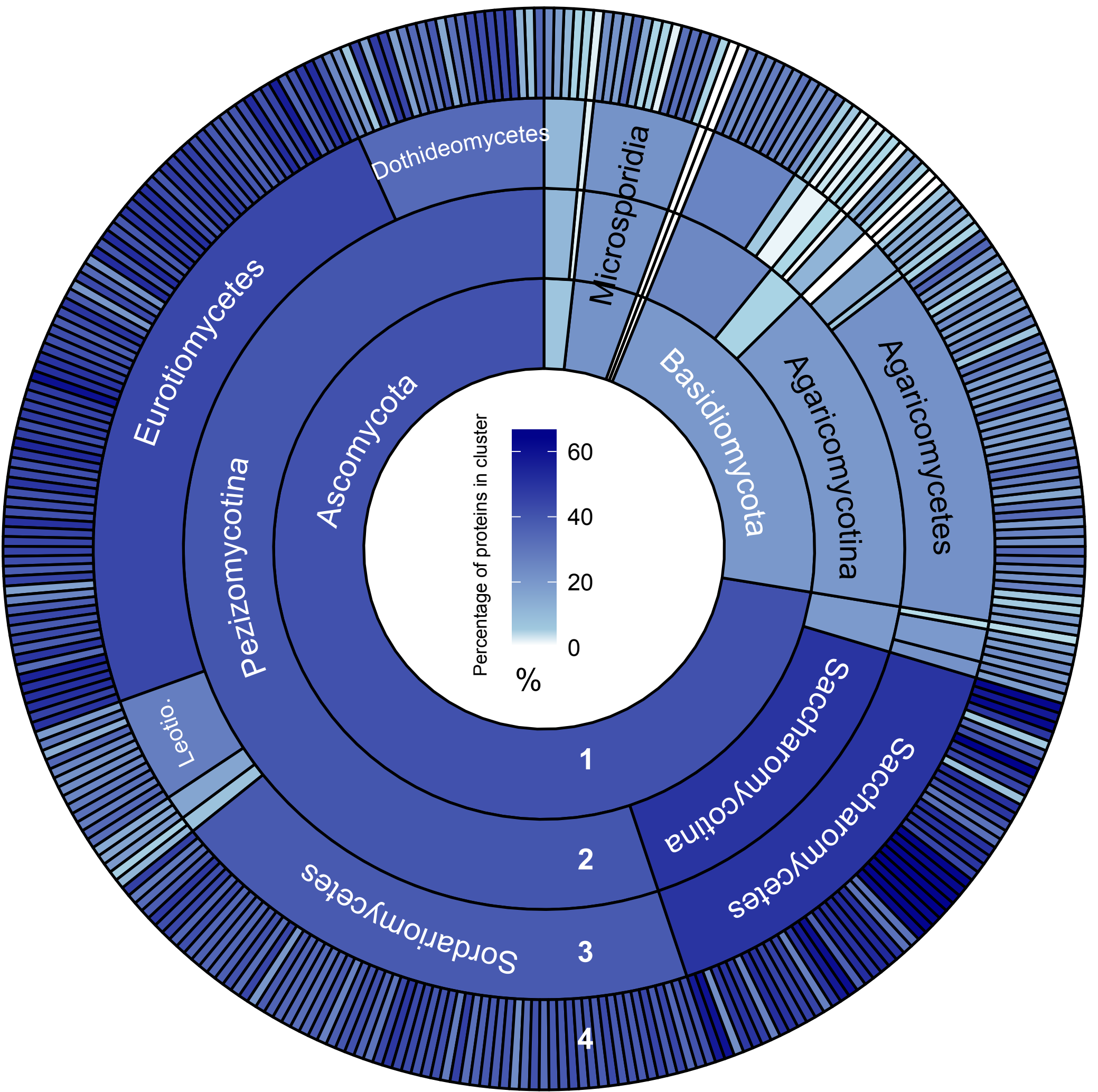
1.- Phylum
2.- Subphylum
3.- Class
4.- Species 
Pseudogymnoascus destructans

Pseudogymnoascus verrucosus

Oidiodendron maius

-Sclerotinia borealis

-Botrytis cinerea

-Sclerotinia sclerotiorum

Phialocephala subalpina

Marssonina brunnea

Rhynchosporium agropyri

Rhynchosporium secalis

Rhynchosporium commune

Glarea lozoyensis

Blumeria graminis

Pestalotiopsis fici

Microdochium bolleyi

Eutypa lata

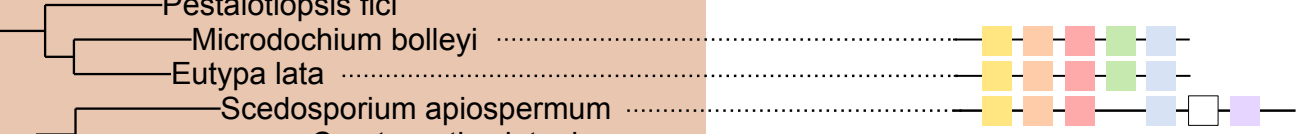

Ceratocystis platani

-Verticillium alfalfae

Verticillium dahliae

- Colletotrichum chlorophyti

Colletotrichum orchidophilum

Colletotrichum fioriniae

-Colletotrichum graminicola

Colletotrichum sublineola

Colletotrichum incanum

Colletotrichum tofieldiae

Colletotrichum orbiculare

Drechmeria coniospora

-Pochonia chlamydosporia

Metarhizium album

Metarhizium acridum

Metarhizium brunneum

Metarhizium robertsii

Metarhizium anisopliae

Metarhizium majus

Metarhizium guizhouense Ustilaginoidea virens

Claviceps purpurea

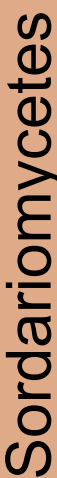

Trichoderma atroviride

-Trichoderma reesei

Trichoderma guizhouense

Trichoderma virens

-Torrubiella hemipterigena

Cordyceps militaris

Beauveria bassiana

Acremonium chrysogenum

Stachybotrys chlorohalonata

Stachybotrys chartarum

Neonectria ditissima

Fusarium oxysporum

Fusarium verticillioides

Fusarium mangiferae

Fusarium fujikuroi

Fusarium proliferatum

-Fusarium avenaceum

Fusarium poae

Fusarium pseudograminearum

Fusarium graminearum

Sordaria macrospora

4 Neurospora tetrasperma

Neurospora crassa

-Podospora anserina

Chaetomium thermophilum

Madurella mycetomatis

Thielavia terrestris

Chaetomium globosum

Myceliophthora thermophila

Grosmannia clavigera

Ophiostoma piceae

Sporothrix schenckii

Sporothrix brasiliensis

Magnaporthe oryzae

Gaeumannomyces graminis

b.-
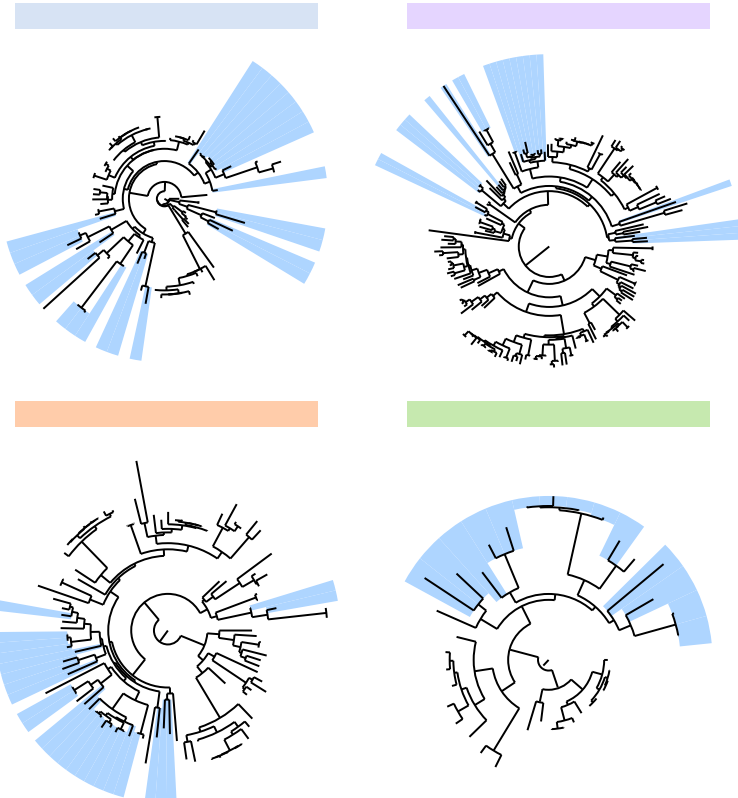

Togninia minima

Valsa mali

-Diaporthe ampelina 
a.- Rasamsonia emersonii (RASEM) -Talaromyces islandicus (TALIS)

-Talaromyces atroroseus (TALAT)

Talaromyces stipitatus (TALSN)

Talaromyces cellulolyticus (TALCE)

Talaromyces marneffei (PENMQ)

Byssochlamys spectabilis (BYSSP)

Penicillium arizonense (PENAR)

Penicillium antarcticum (PENAN)

Penicillium nordicum (PENNO)

Penicillium freii (PENFR)

Penicillium polonicum (PENPO)

Penicillium solitum (PENSO)

Penicillium expansum (PENEN)

Penicillium italicum (PENIT)

Penicillium digitatum (PENDI)

Penicillium roqueforti (PENRO)

Penicillium nalgiovense (PENNA)

Penicillium rubens (PENCH)

-Penicillium flavigenum (PENFL)

Penicillium vulpinum (PENVU)

Penicillium steckii (PENST)

Penicillium oxalicum (PENOX)

Penicillium brasilianum (PENBR)

Penicillium subrubescens (PENSU)

Aspergillus cristatus (ASPCR)

Aspergillus ruber (ASPRU)

- Aspergillus clavatus (ASPCL)

Neosartorya udagawae (NEOUD)

Aspergillus udagawae (ASPUD)

Aspergillus fumigatus (ASPFU)

Neosartorya fischeri (NEOFI)

Aspergillus terreus (ASPTE)

Aspergillus parasiticus (ASPPA)

Aspergillus flavus (ASPFL)

Aspergillus oryzae (ASPOR)

Aspergillus nomius (ASPNO)

Aspergillus bombycis (ASPBO)

Aspergillus niger (ASPNI)

Aspergillus kawachii (ASPKA)

Aspergillus luchuensis (ASPLU)

Aspergillus rambellii (ASPRA)

Aspergillus ochraceoroseus (ASPOC)

Aspergillus calidoustus (ASPCA)

Aspergillus nidulans (EMENI)

-Paracoccidioides brasiliensis (PARBR)

-Blastomyces dermatitidis (BLADE)

Histoplasma capsulatum (AJECA) -Arthroderma otae (ARTOT)

Microsporum gypseum (MICGY)

Trichophyton interdigitale (TRIIN)

Trichophyton tonsurans (TRITO)

Trichophyton equinum (TRIEQ)

Trichophyton violaceum (TRIVI)

Trichophyton soudanense (TRISO)

Trichophyton rubrum (TRIRU)

Arthroderma benhamiae (ARTBE)

Trichophyton verrucosum (TRIVE)

Uncinocarpus reesii (UNCRE)

Coccidioides immitis (COCIM)

Coccidioides posadasii (COCPO)

Phaeomoniella chlamydospora (PHACH)

Endocarpon pusillum (ENDPU)

- Phialophora attae (PHIAT)
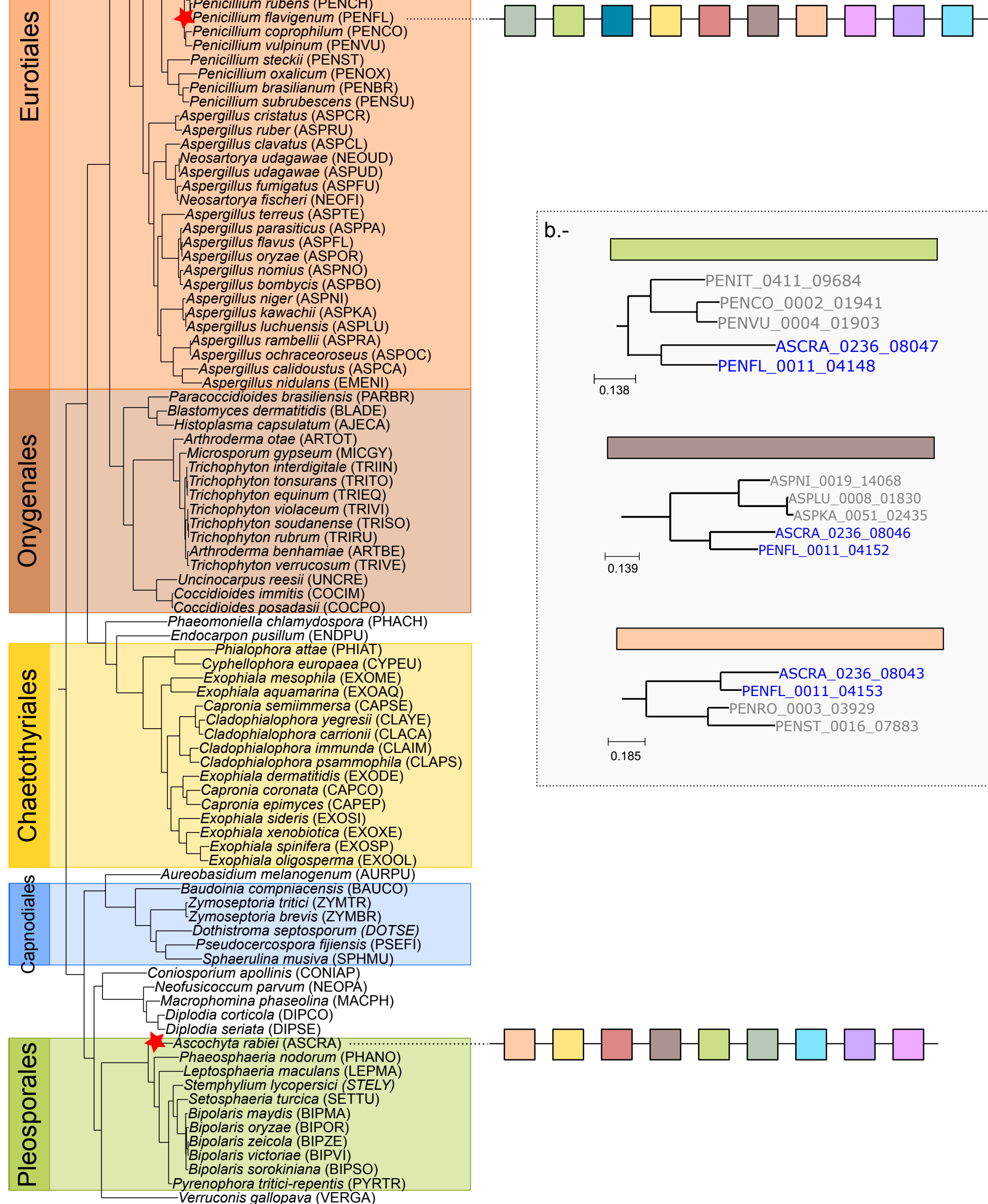

b.-
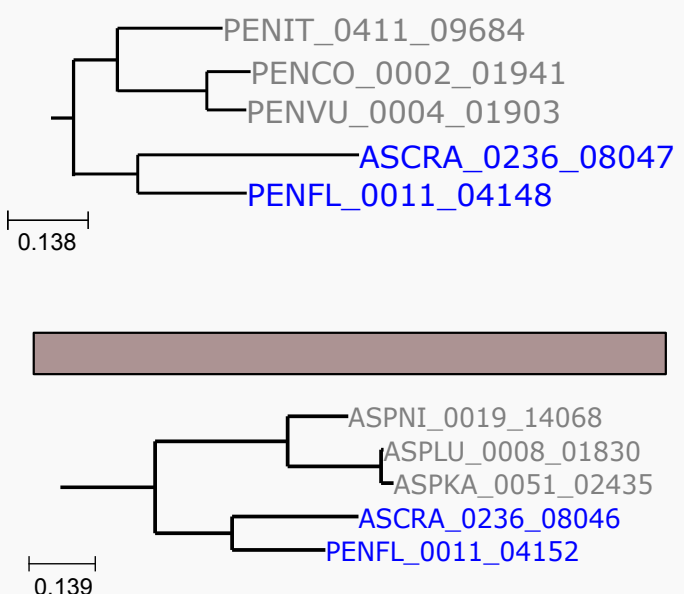

ASCRA $0236 \quad 08043$

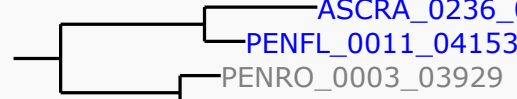

PENST_0016_07883

0.185

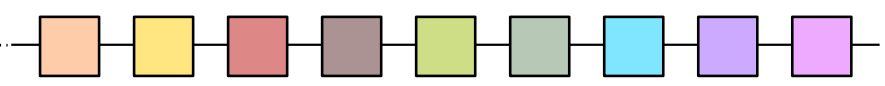

Pyrenophora tritici-repentis (PYRTR) 
a.-

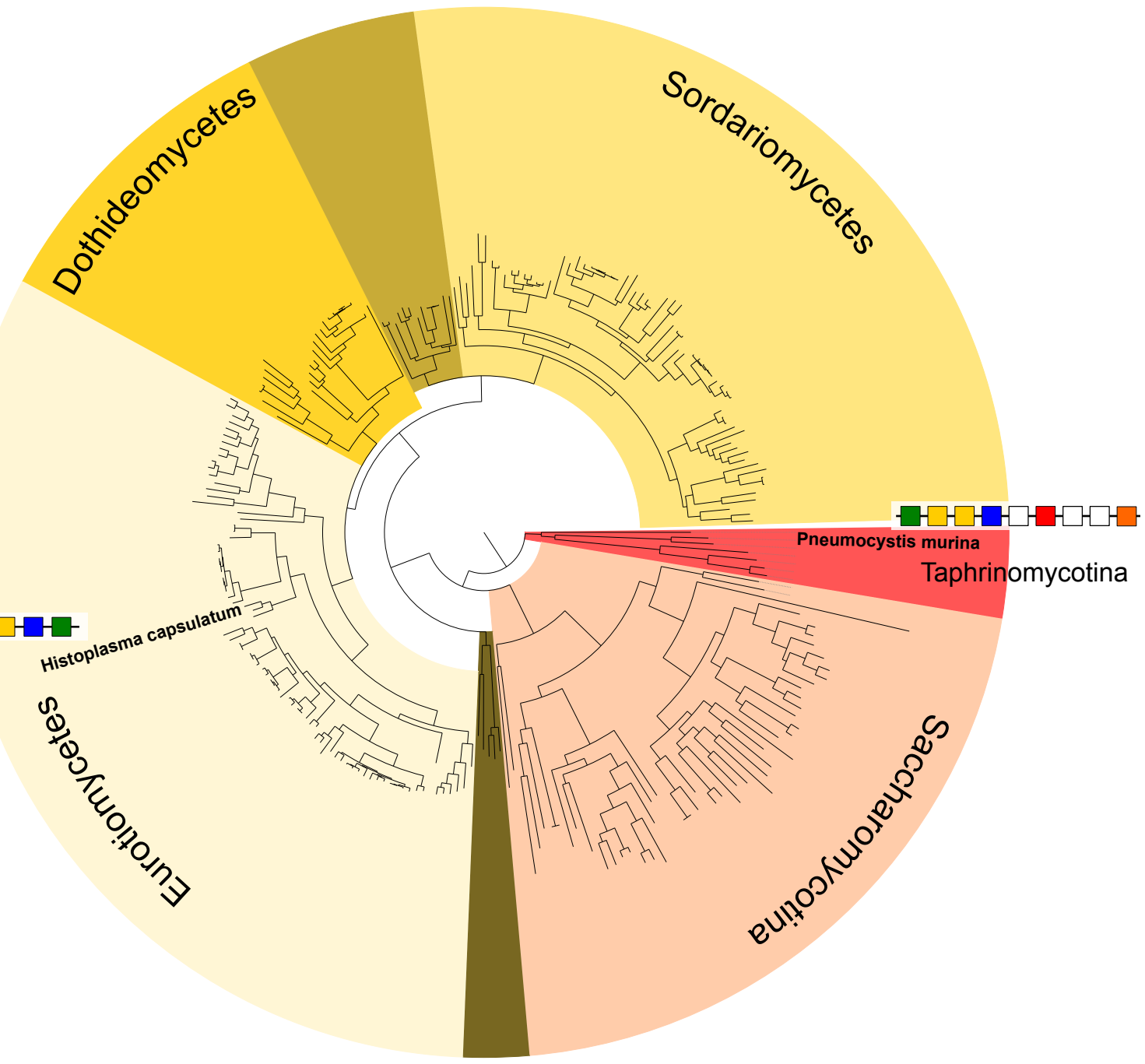

b.-
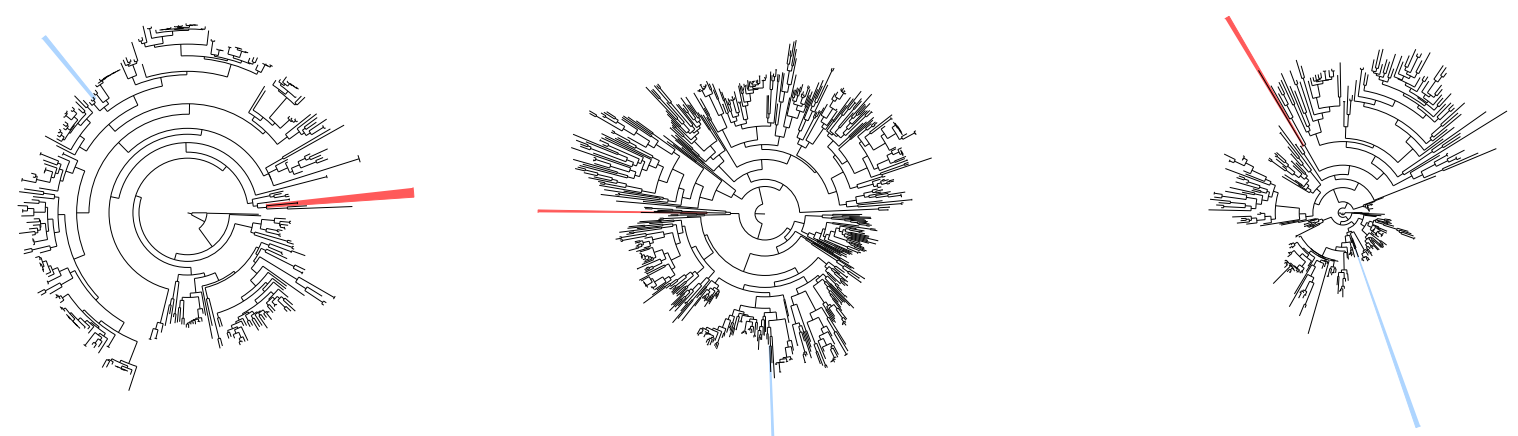
a)

Vertical evolution

Convergent evolution

All cluster families

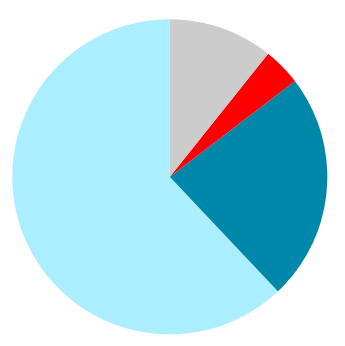

Cluster families secondary metabolism

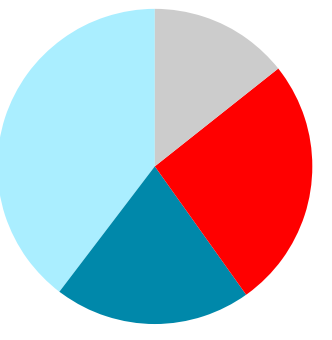

Widespread cluster families

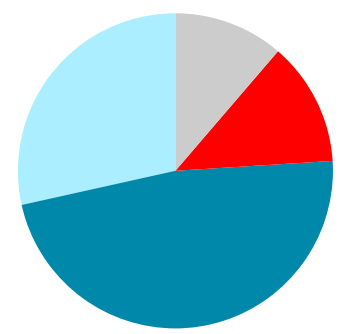

Cluster families secondary metabolism with known function

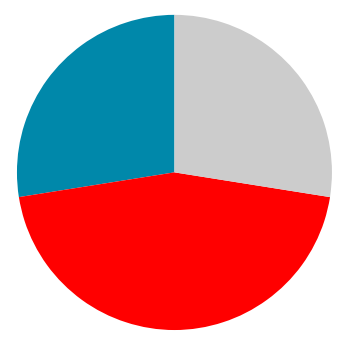

b)

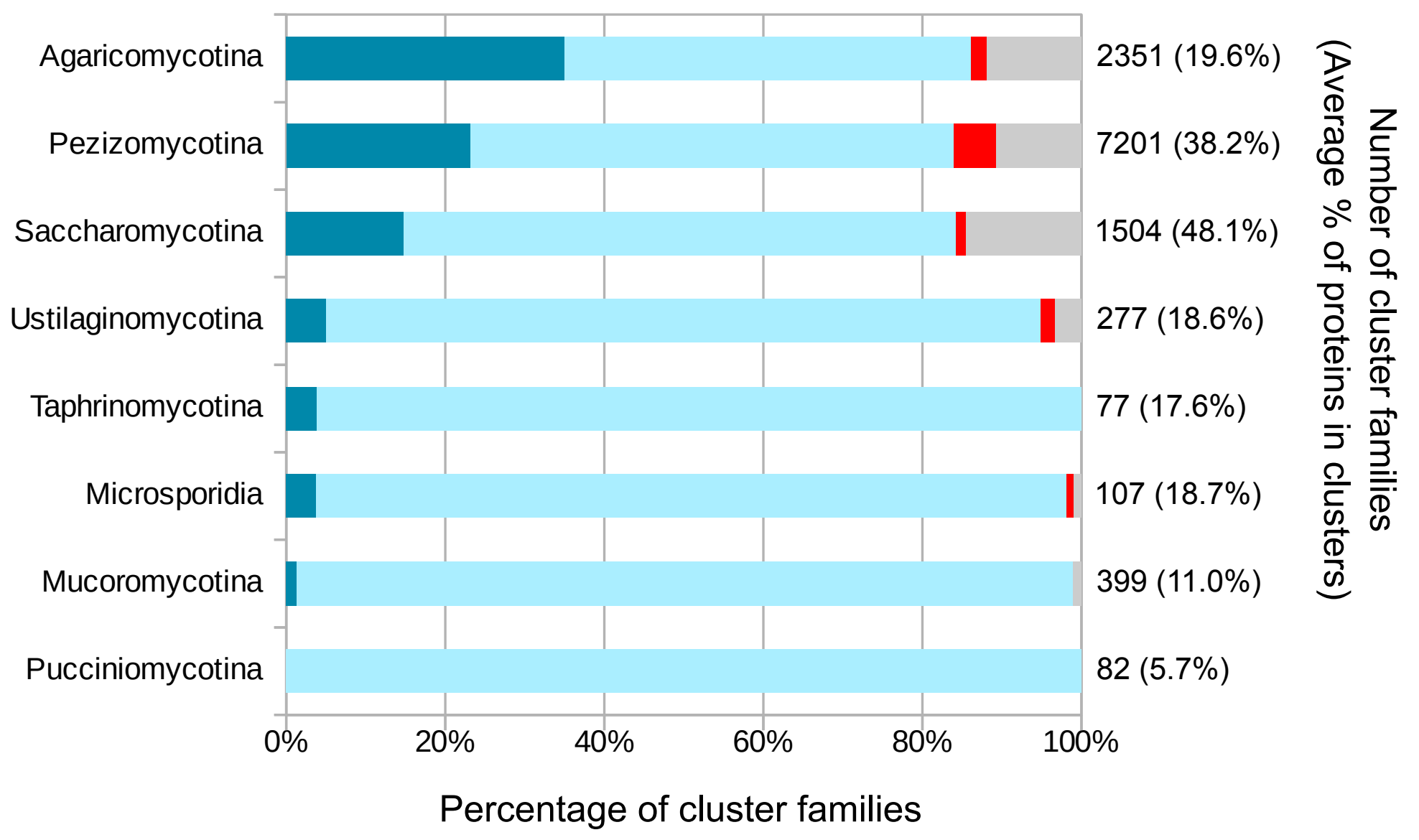


Supplementary information for: Evolutionary and functional patterns of shared gene neighborhood in fungi 


\section{Supplementary Discussion}

Comparison of clustering methods in a set of Dothideomycetes species.

Ohm and collaborators ${ }^{26}$ provided two sets of conserved clusters, one from eight Pleosporales species of which six were in our dataset (Bipolaris maydis, Bipolaris sorokiniana, Leptosphaeria maculans, Phaeosphaeria nodorum, Pyrenophora tritici-repentis and Setosphaeria turcica) (OhmP) and one from six mycosphaerella species of which three were in our dataset (Dothistroma septosporum, Sphaerulina musiva and Zymoseptoria tritici) (OhmM). Results from Ohm et al. were compared to results obtained from EvolClust when applied on the large dataset (341 species) and to EvolClust run independently on the shared species. The results were congruent in both cases. We also ran Gecko ${ }^{27}$ on these two datasets. Gecko3 was run with the following parameters: -d 10 -q 2 -s 5. The comparison between the first set (OhmP) showed similar results in terms of total number of clusters (OhmP: 2778 clusters and EvolClust 2751 clusters), yet in OhmP clusters are grouped into 502 families whereas EvolClust divided them in 714 families. Only four of the clusters were predicted with exactly the same boundaries between the two sets, yet in 50\% of cases the clusters predicted in OhmP were a subset of the EvolClust predicted cluster. If we consider overlaps of at least $50 \%$ of the cluster, $88 \%$ of the clusters in OhmP were found in the EvolClust dataset. The smaller dataset (OhmM) is curious because it contains much less conserved blocks than the previous dataset (58 versus 502) whereas EvolClust does not decrease the dataset so much (259 vs 714). Gecko3 produces results more similar to EvolClust and predicts 284 cluster families. This may be influenced by the fact that clusters predicted by $\mathrm{Ohm}$ et al. are required to be present in $70 \%$ of the species in the group whereas EvolClust and Gecko3 did not have such a requirements. 98\% of the clusters found in OhmM were present in the EvolClust dataset though none of them were identical. Gecko3 produced more similar results with 289 clusters with identical predictions and an additional 250 clusters being a subset of EvolClust (this represents $72.8 \%$ of all clusters).

Of note is the percentage of proteins found in clusters for these datasets. In OhmP $48.7 \%$ of the genes are found in cluster whereas EvolClust predicts that $38.0 \%$ of the proteins are in cluster. OhmM has only $7.1 \%$ of proteins in cluster. EvolClust and Gecko3 present higher percentages standing at $13.3 \%$ and $17.1 \%$ respectively.

\section{Elements influencing gene cluster predictions}

Algorithmic and genomic factors affecting EvolClust predictions.

Maximal cluster size 
As seen in the analysis above, the number of clusters predicted by EvolClust is in line with similar algorithms. Still, like in most algorithms, the parameters used when executing EvolClust will have a direct effect on the number of predicted clusters. One such parameter is the maximum cluster size. The upper level of 35 exceeds the size of most known metabolic gene clusters yet, the pneumocandin gene cluster for instance is predicted to have 31 proteins and other clusters such as aflatoxin, fumonisin or echinocandin B have over 20 proteins. An upper threshold is necessary because the longer the cluster the more likely it is to overwhelm the threshold distinguishing it from background gene order conservation.xWe can estimate how results would change with a smaller maximum cluster size. Take for instance a pair of species, in this case Aspergillus flavus and Penicillium expansum. If we use the maximum size of 35, we predict 124 clusters which include between $16 \%$ and $20 \%$ of their respective genomes. With a limit of 15 proteins on the other hand we detect 46 clusters which represents between $3.8 \%$ and $4.6 \%$ of their genomes. So, we could force the detection of a smaller percentage of proteins in cluster, but that would come at the cost of not being able to detect longer clusters. We thus in this study we decided to use 35 , a threshold slightly longer than the maximal size found in known clusters.

\section{Significant conservation threshold}

Another element influencing the prediction of clusters is the way thresholds that distinguish between background gene order conservation and gene clusters are calculated. EvolClust implements different ways to calculate the thresholds: based on the standard deviation (1, 2 or 3 times the standard deviation of the average conservation of gene order between two species) or based on the percentage of clusters that would pass the threshold $(75 \%$ or $90 \%)$. In the same example used above, we predicted 39 clusters when using as threshold a measure calculated by using three times the standard deviation. Whereas if we use the threshold that allows $10 \%$ of the clusters to be included 148 clusters will be included. In this study we opted to use 2 -standard deviations from the average as a threshold, as this is a standard threshold to define significance in normally-distributed data.

\section{Phylogenetic distribuion threshold}

Another consideration is that EvolClust, unlike Gecko3 and the algorithm designed by Ohm et al., does not offer the possibility to limit clusters by the number of species they appear in. Still it is not difficult to see how such a limit would influence the number of clusters. If we only consider clusters found in at least five species, we reduce the number of detected clusters to 6272 $(\sim 50 \%)$. However, we were interested in detecting sparsely distributed clusters as well, and therefore we decided not to set this threshold. 
In this study we decided to use a single genome to represent each species. This may influence the results if gene content (and order) differs largely between different strains of the same species. While this is true and in the future it would be an interesting field of study, the fact is that nowadays only few species with several sequenced strains are available. Therefore we consider that with the current availability of data, using alternative strains would have not significantly altered the overall results of our analyses.

\section{Fast evolving genes and homology detection}

If a gene evolves fast enough that it is not detected by the blast-search step, it may not be included in a cluster. Still, the flexibility provided by EvolClust to allow non-homologous proteins in between homologous proteins likely minimizes this effect. We only would expect this to matter when these kind of fast evolving genes are at the edge of the cluster and even so, in such a large dataset, this is likely anecdotic.

\section{Differential rates of genome re-arrangements}

Something that may influence more our results is the presence of large groups of species with an overly conserved gene order conservation or affected by events that limit gene order shuffling such as mesosynteny. Anything influencing the free movement of genes in the different genomes is likely to confound an algorithm based on observed and expected levels of gene order conservation. However, this effect is expected to be mitigated by the use of EvolClust of different thresholds for each pairwise comparison, being in each case dependent on the observed distributions of gene order conservation. This would lead to higher thresholds in species that evolve slower in terms of gene order.

\section{Highly duplicated gene families}

Another confounding factor afecting gene cluster prediction is the presence of highly duplicated families, which have a higher probability that single-gene families to have, by chance, two of their respective members in close proximity. To test how prevalent this effect may be, fwe randomly shuffled the gene tags for each species and then, for each cluster we searched if it was present in this randomly shuffled genome. This was repeated a hundred times for each species. Out of the 341 species used in this analysis only in 31 did we detect some cluster in the randomized datasets. In most of those (24) three or less clusters were detected in one of the repetitions. The most concerning case belonged to the polyploid Rhizopus delemar that had 59 potentially random clusters (16\% of the clusters detected in this species) and this can likely be attributed to the higher than normal rate of duplicated in this polyploid. Outside such cases, we expect that the effect of duplicated genes in cluster prediction is minimal.

\section{Genome assembly fragmentation}

Although we selected for our analysis only genome assemblies with less than 2000 contings, different levels of assembly fragmentiation could affect gene cluster prediction. To assess whether assembly fragmentation -within the levels found in our dataset- could affect gene clusterprediction and the assignment to evolutionary events we took the 50 best assembled 
genomes in our dataset. This set of species included 30 Saccharomycotina (60\%), 16 Pezizomycotina (32\%), 2 Taphrinomycotina (4\%) and 2 Microsporidia (4\%). Therefore, as expected, the taxonomic diversity is quite different from the whole dataset. For the selected species, we simulated different levels of fragmentation. So, each of the 50 genomes was randomly assigned a number of genes per contig that was taken from the original dataset and then the genome was fragmented until it had the same number of genes per contig. This process was repeated 10 times so that we obtained 10 different datasets. We used EvolClust to predict cluster families in these 11 ( 1 original plus 10 fragmented) datasets and we observed that the fragmented datasets had a larger amount of predicted cluster families, which is expected as fragmentation can divide a cluster in two parts. The original dataset predicted 2020 cluster families whereas for the fragmented datasets we have an average number of cluster families of 2142 (standard deviation +24 ). Given the predicted cluster families we used evolclassifier to obtain the evolutionary mechanisms by which each family has evolved. We found that $95.4 \%$ of the clusters of the original dataset followed vertical evolution. In the fragmented datasets we have an average number of $95.6 \%$ (standard deviation $+0.3 \%$ ) of cluster families following vertical evolution. Convergent evolution was found in $3.9 \%$ of the cluster families in the original dataset and in 3.9\% (standard deviation +0.3 ) in the fragmented datasets. Finally horizontal gene transfer events were found in $0.7 \%$ of the original cluster families and in $0.5 \%$ (standard deviation $+0.06 \%$ ). So fragmentation does not seem to affect the prediction of evolutionary mechanisms. And therefore, while it is likely that in our original dataset the number of clusters is larger than would be expected due to fragmentation, we still expect to have the same distribution of evolutionary events. 
Supplementary figure 1

\section{Pezizomycotina}

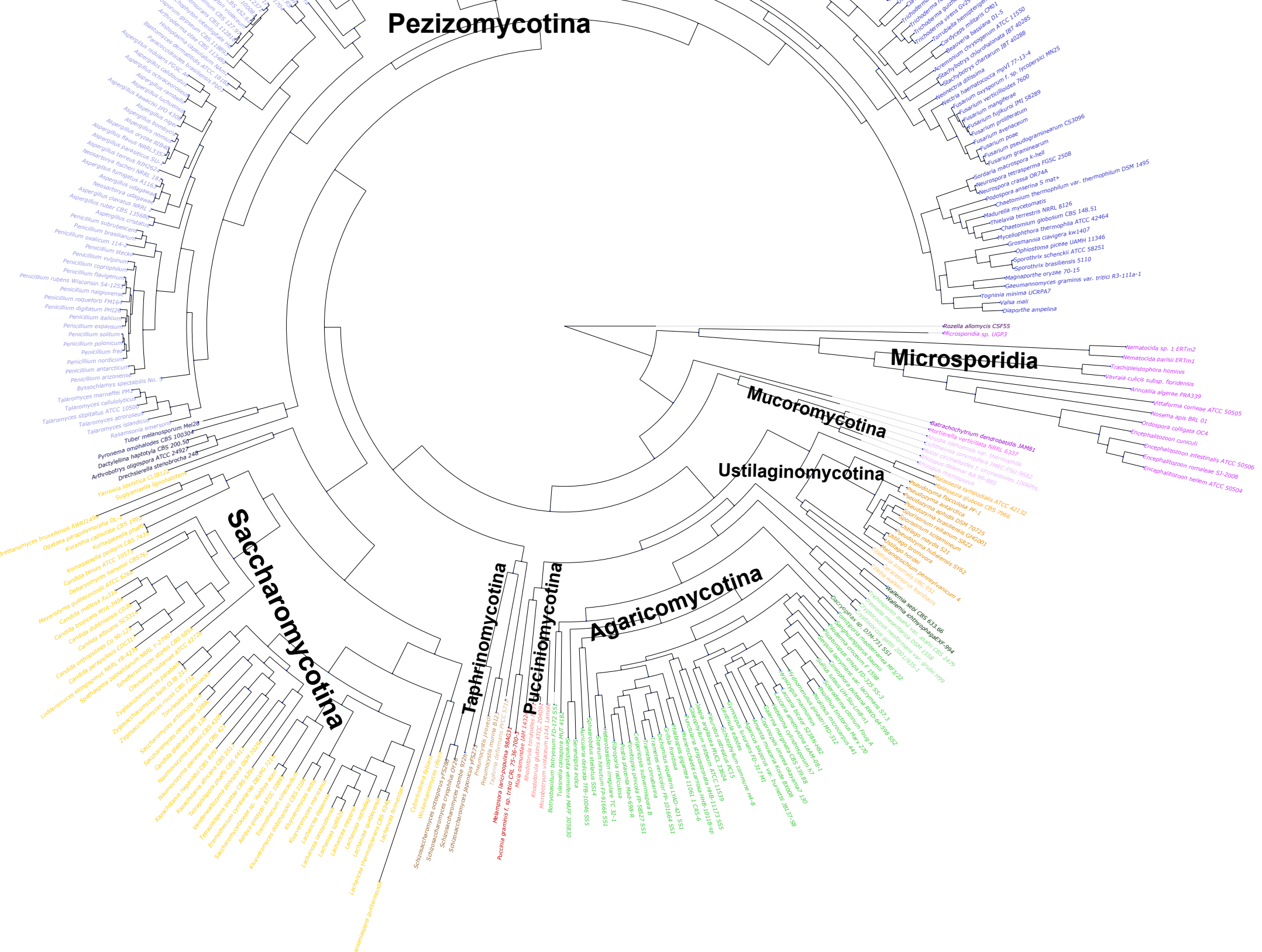




\section{Supplementary figure 2}

a

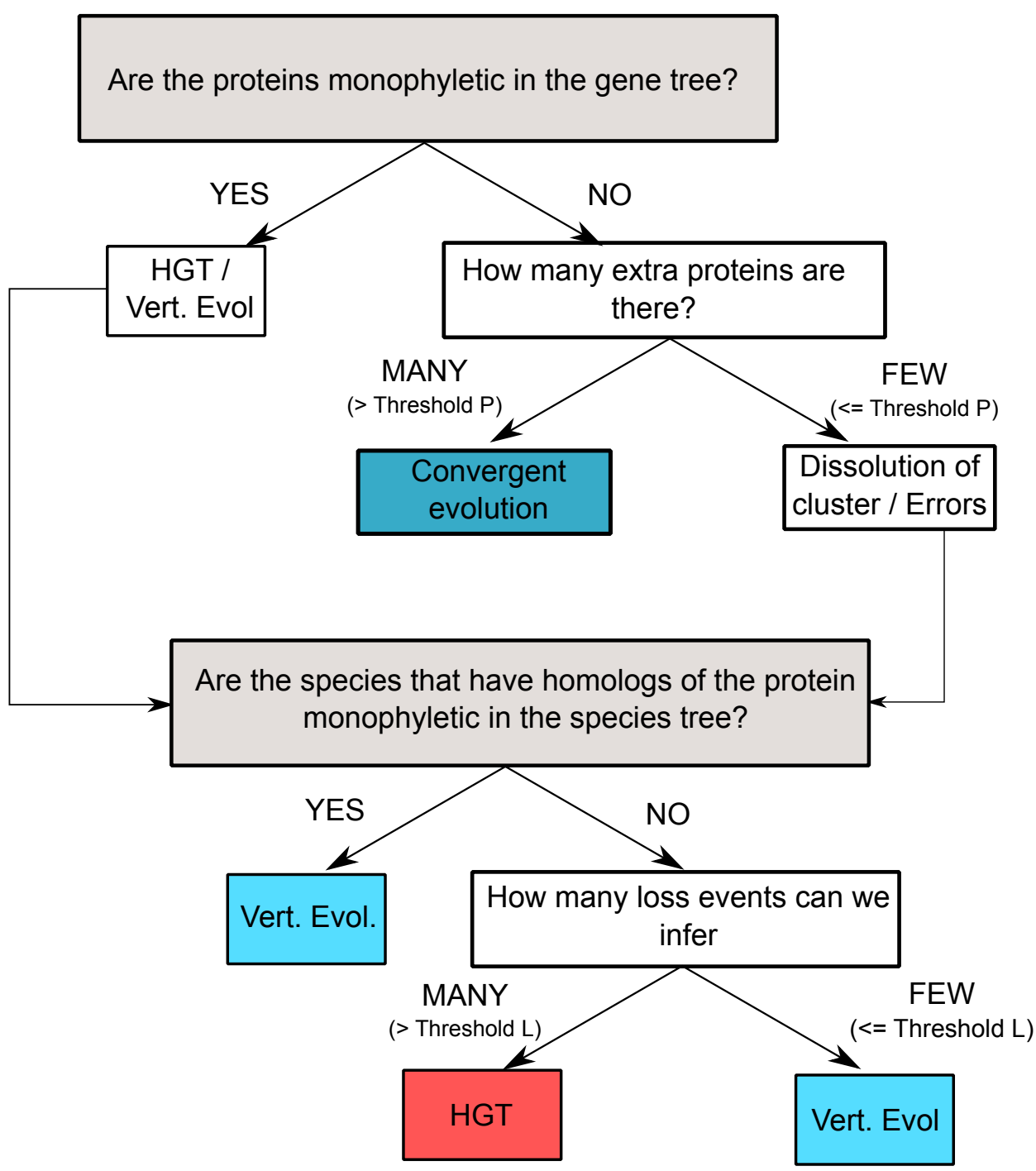

b

GFs
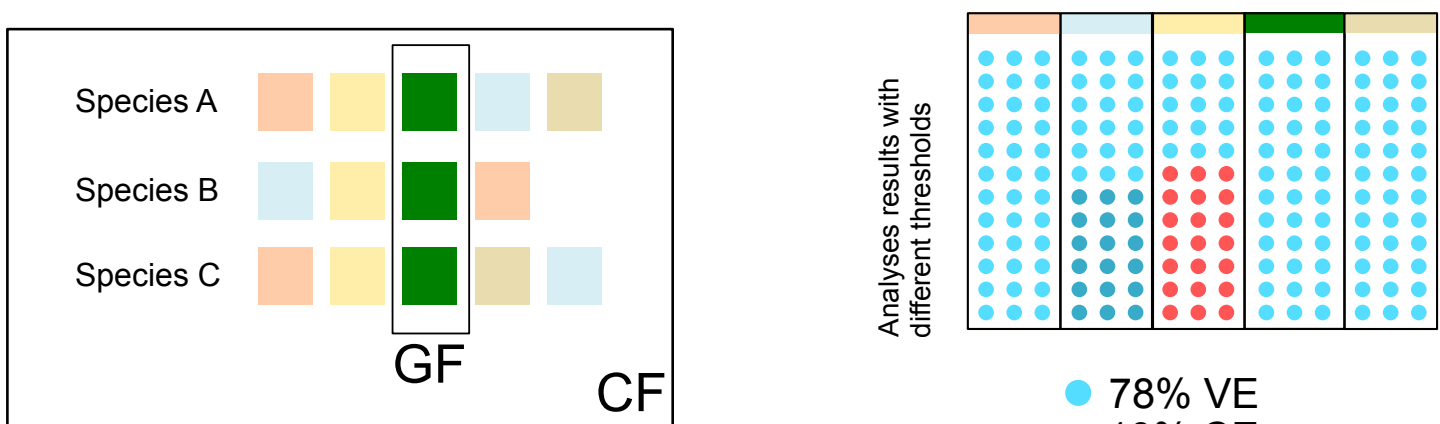

$78 \%$ VE

$10 \%$ CE

$12 \%$ HGT 


\section{Supplementary figure 3}

a
Vertical evolution
Undecided
- Convergent evolution
Horizontal gene transfer

All cluster families

Widespread cluster families
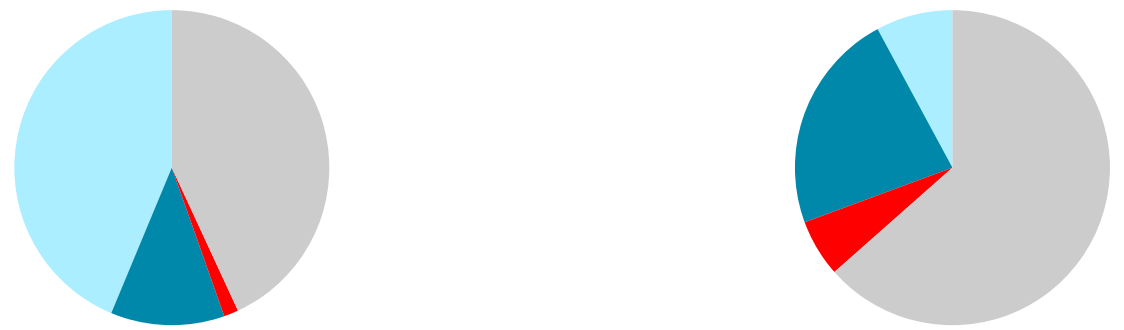

Cluster families secondary metabolism

Cluster families secondary metabolism with known function
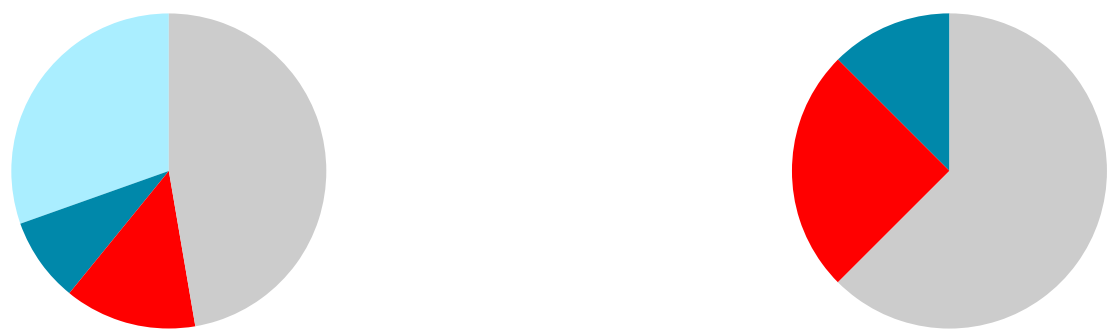

b

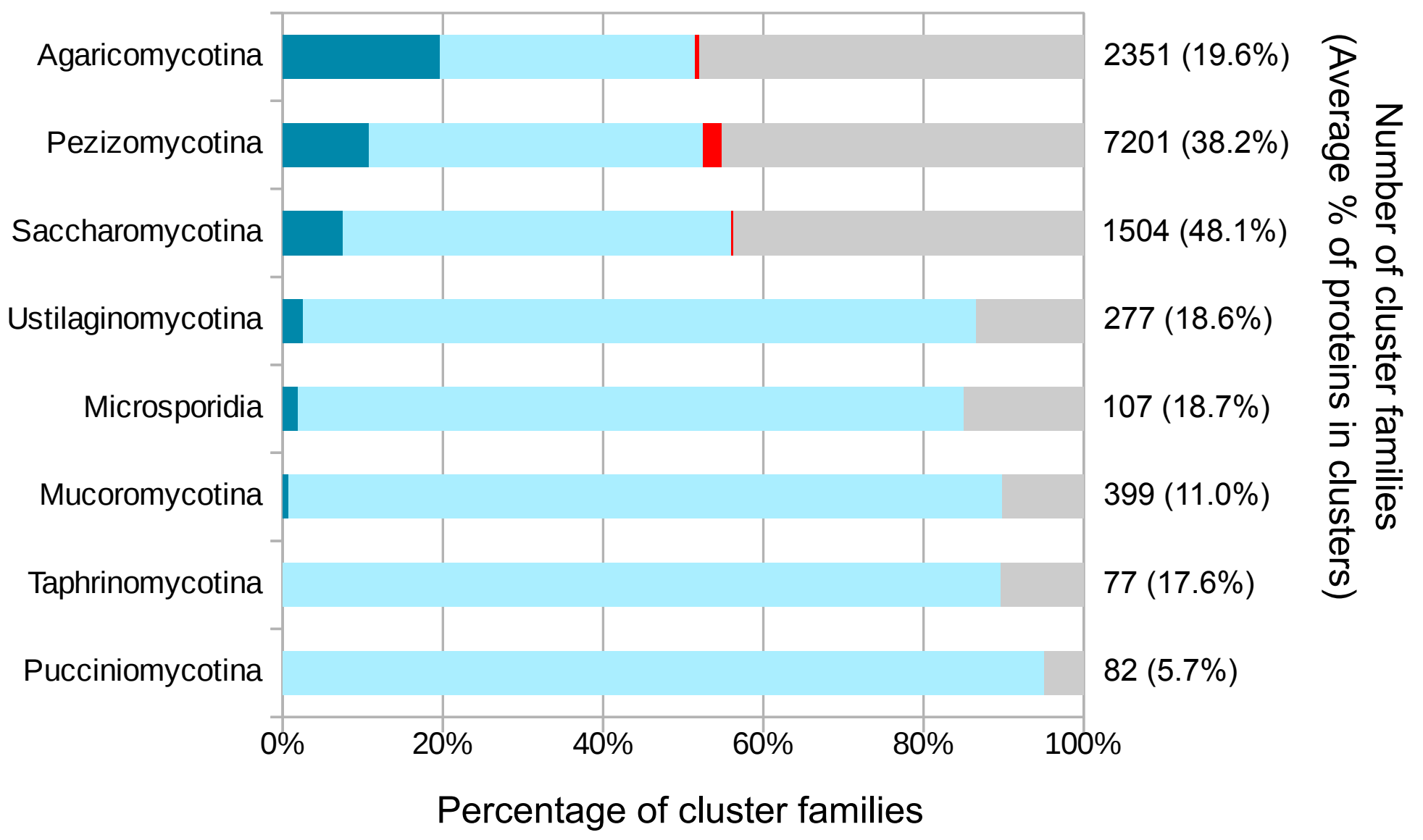


Supplementary figure 4
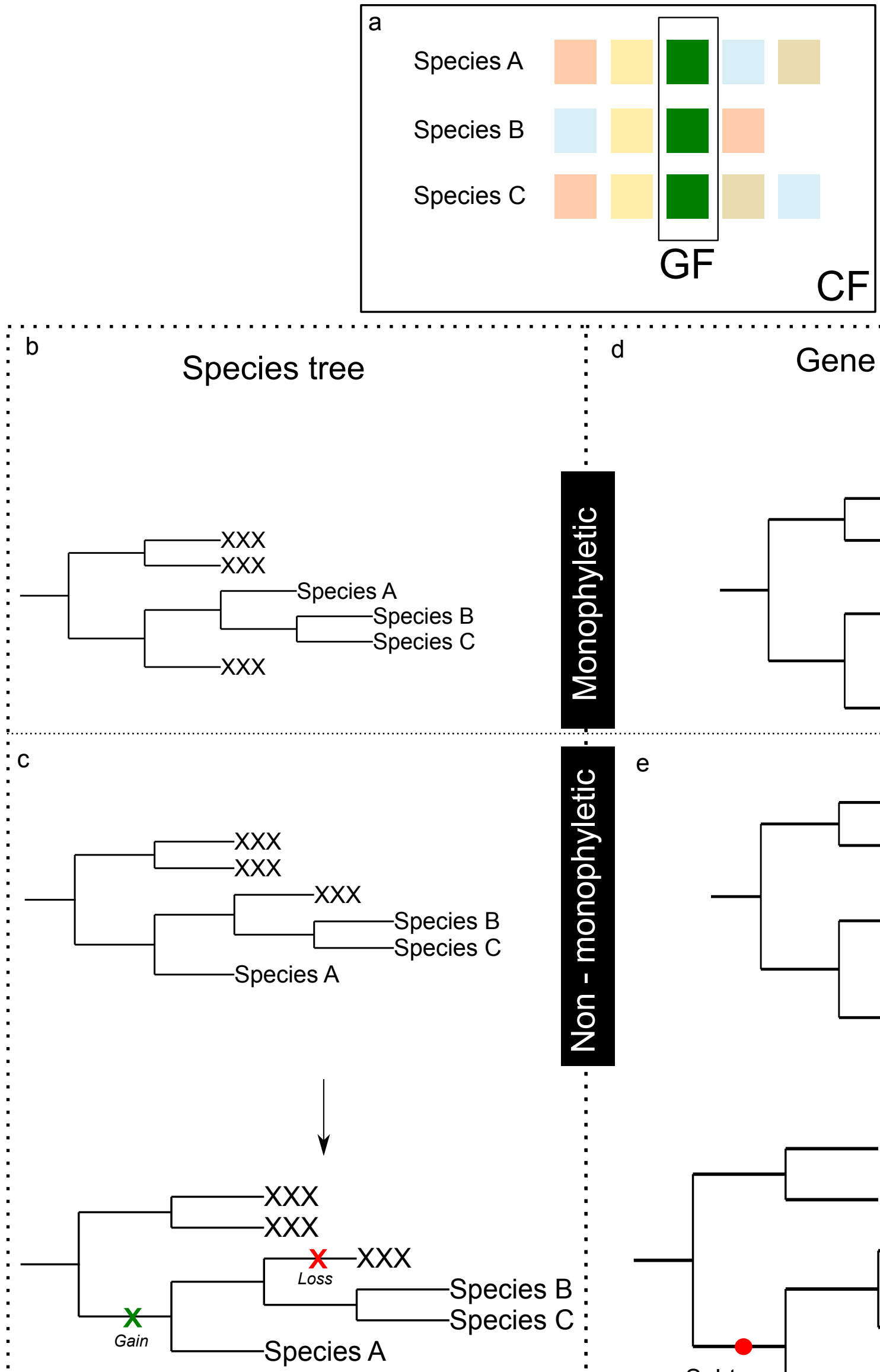

d

Gene tree

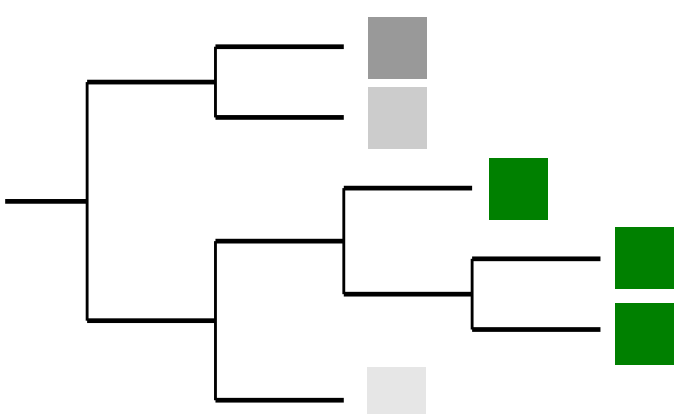

e
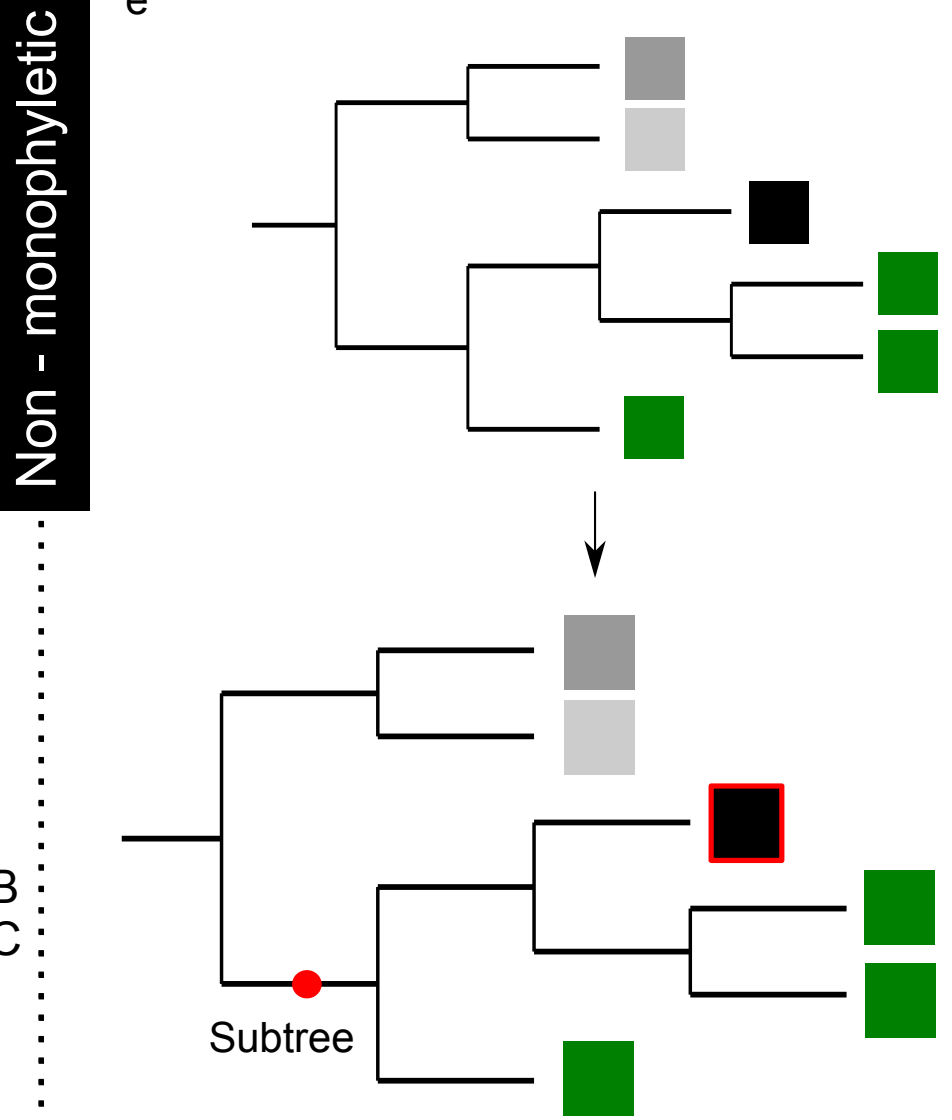

Threshold L: Number losses 


\section{Legends for Supplementary Figures and Tables.}

Supplementary Figure 1: Species tree containing the relationships among 341 species used in this study. The tree was reconstructed using fasttree and a concatenated alignment of 442 protein families.

Supplementary Figure 2: a.- Main EvolClassifier algorithm. EvolClassifier classifies the different homologous families in a cluster family into different evolutionary categories. b.- On the right there is a graphical representation of a cluster family (CF) with five different gene families (GF) represented by squares of different colours. On the left is a graphical representation of the decision making process. For each GF, represented in a rectangle, different thresholds are used to obtain the evolutionary mechanism using the decision tree above. Each such analysis is represented by a dot. The colour of the dot indicates the decision made, if more than $50 \%$ of the dots have the same decision then this decision is assigned to the GF as indicated by the rectangle found below the dots. If no evolutionary mechanism reaches $50 \%$ of the analyses then the GF is left as undecided. The final assignation of the cluster is given by the majority of the GF evolutionary mechanisms as long as they are higher than $50 \%$.

Supplementary Figure 3: Pie charts representing the percentage of clusters that evolved primarily through a given evolutionary mechanism in dataset75. Charts are build for all cluster families, the widespreadCF subset, the group of cluster families predicted to encode secondary metabolism gene clusters, and those of the last category that have a known function. B.- Percentage of clusters that follow each of the three considered evolutionary mechanisms in each subphylum for dataset75. Numbers on the right of the graph represent the total number of cluster families that map within each subphylum and the average percentage of proteins in cluster in those species.

Supplementary Figure 4: Detail on the parameters calculated to decide the evolutionary model. a.Graphical representation of a cluster family $(\mathrm{CF})$. Each row represents a species that contains the cluster, each square represents a protein-coding gene that forms part of the cluster. Colours define homologous gene families (GF). b.- Example of a monophyletic species tree. c.- Example of a nonmonophyletic species tree and how loss events are inferred. The cluster is assumed to be gained at the common ancestor of all species that have it - represented in a green X. In the example only one loss is found - represented with a red X. d.- Example of a gene tree where all genes belonging to the same GF are monophyletic. e.- Example of a gene tree where genes of the same GF are not monophyletic. The number of additional genes in the subtree, marked with a red circle, that contains all GF can be computed. In this case only one additional protein is present represented in a black square with a red rim.

Supplementary Table 1: Species information. List of 341 species used in the study. Columns indicate, from left to right: species name, species code, BioProject at NCBI, and the taxonomic categories of the species as found in NCBI taxonomy and used in the study. If a category is filled with a dash it means the category does not exist for that species.

Supplementary Table 2: List of predicted clusters. Each cluster is indicated by a \# symbol followed by a code that starts with CF and a 6-digits code. The following rows indicate the clusters belonging to each family. For each cluster, the first column indicates the species code, when an underscore followed by a number is present in the species name it indicates the duplication of the cluster, the second shows the species name and the last contains the list of proteins in that are part of the cluster. Codes refer to Locus tags as provided by NCBI. 
Supplementary Table 3: GO term enrichment for proteins in cluster. Comparison is done between proteins that are found in the clusters $(n=1,100,163$ proteins $)$ as compared to the complete protein database $(\mathrm{n}=3,421,149$ proteins). Enrichment was considered with an adjusted $\mathrm{p}$-value $<$ 0.001 (two-sided). Table is divided in three parts: Biological process, Cellular component and Molecular function. Each part contains three columns: the GO ID, the GO description and the last column indicates the $\log 10$ of the adjusted p-value as provided by FatiGO.

Supplementary table 4: GO term enrichment for proteins in CF2. Comparison is done between proteins in CF2 $(n=251,977$ proteins) versus the list of all proteins in cluster $(n=949,693$ proteins). Proteins of Saccharomycotina, Mucoromycotina and Microsporidia were excluded from the dataset. Enrichment was considered with an adjusted p-value $<0.001$ (two-sided). Table has the same distribution as Supplementary Table 3.

Supplementary Table 5: List of families that contain a transcription factor. Only families where at least half of the clusters contain a transcription factor are included. For families of 2 or 3 members, the transcription factor has to be present in at least two of the clusters.

Supplementary Table 6: GO term enrichment for transcription factors. Comparison is done between proteins in cluster families that contain a transcription factor $(n=206,091)$ versus the list of proteins found in all clusters $(\mathrm{n}=1,100,163$ proteins). Enrichment was considered with an adjusted p-value $<0.001$ (two-sided). Table has the same distribution as Supplementary Table 3.

Supplementary Table 7: List of evolutionary events that shaped each cluster family. Column 1 indicates the cluster name as in Supplementary Table 2. Columns 2 and 3 indicate the number of clusters and the number of species in the cluster family, respectively. Columns 4 and 5 indicate the taxonomy category of the common ancestor of each cluster family and its taxonomic level. Column 6, 7 and 8 indicate the percentages of analyses that support each of the three possible events VE (vertical evolution), CE (convergent evolution) and HGT (horizontal gene transfer). Column 9 indicates the predicted number of re-arrangements calculated by MGR when possible. Column 10 and 11 indicate the major evolutionary event for the cluster family based on two different thresholds: $50 \%$ and $75 \%$.

Supplementary Table 8: Mapping of the COG terms to each cluster family. First column indicates the cluster family as seen also in supplementary table 3. Column 2 indicates the COG categories associated to the cluster family. The rest of the columns indicate in which percentage each category is present in the cluster family.

Supplementary Table 9: Enrichment of COG terms in groups of families that evolved through different evolutionary mechanisms according to the general annotation. First column indicates the COG functional ID, the second column indicates the name of the COG functional ID, the third column indicates in which category the COG functional ID can be found and finally the two last columns indicate whether each dataset is enriched in families that evolved through vertical inheritance (VE), horizontal gene transfer (HGT) or it is not enriched in any group of cluster families (-). Enrichment was considered with an adjusted p-value $<0.001$ (two-sided).

Supplementary Table 10: GO term enrichment for proteins in clusters that underwent vertical evolution (dataset50). Comparison is done between proteins in cluster families that evolved through vertical inheritance in dataset50 $(n=830,939)$ versus the list of proteins found in all clusters $(\mathrm{n}=1,100,163$ proteins $)$. Enrichment was considered with an adjusted $\mathrm{p}$-value $<0.001$ (two-sided). Table has the same distribution as Supplementary Table 3. 
Supplementary Table 11: GO term enrichment for proteins in clusters that underwent horizontal gene transfer (dataset50). Comparison is done between proteins in cluster families that evolved through horizontal gene transfer in dataset50 $(\mathrm{n}=17,934)$ versus the list of proteins found in all clusters $(\mathrm{n}=1,100,163$ proteins). Enrichment was considered with an adjusted p-value < 0.001 (two-sided). Table has the same distribution as Supplementary Table 3.

Supplementary Table 12: GO term enrichment for proteins in clusters that underwent convergent evolution (dataset50). Comparison is done between proteins in cluster families that evolved through horizontal convergent evolution in dataset50 $(\mathrm{n}=178,317)$ versus the list of proteins found in all clusters $(n=1,100,163$ proteins). Enrichment was considered with an adjusted p-value $<0.001$ (two-sided). Table has the same distribution as Supplementary Table 3.

Supplementary Table 13: GO term enrichment for proteins in clusters that underwent vertical evolution (dataset75). Comparison is done between proteins in cluster families that evolved through vertical inheritance in dataset75 $(\mathrm{n}=500,938)$ versus the list of proteins found in all clusters $(n=1,100,163$ proteins). Enrichment was considered with an adjusted $\mathrm{p}$-value $<0.001$ (two-sided). Table has the same distribution as Supplementary Table 3.

Supplementary Table 14: GO term enrichment for proteins in clusters that underwent horizontal gene transfer (dataset75). Comparison is done between proteins encoded in cluster families that evolved through horizontal gene transfer in dataset75 $(n=5,669)$ versus the list of proteins found in all clusters $(n=1,100,163$ proteins). Enrichment was considered with an adjusted p-value $<0.001$ (two-sided). Table has the same distribution as Supplementary Table 3.

Supplementary Table 15: GO term enrichment for proteins in clusters that underwent convergent evolution (dataset75). Comparison is done between proteins in cluster families that evolved through horizontal convergent evolution in dataset $75(\mathrm{n}=57,783)$ versus the list of proteins found in all clusters ( $\mathrm{n}=1,100,163$ proteins). Enrichment was considered with an adjusted $\mathrm{p}$-value $<0.001$ (two-sided). Table has the same distribution as Supplementary Table 3.

Supplementary Table 16: List of detailed evolutionary events assigned for each cluster family. First column indicate the cluster family, second and third column indicate the evolutionary events assigned for dataset50 and dataset 75 in the detailed evolutionary analysis. Note that multiple events can be assigned to one cluster family.

Supplementary Table 17: List of 132 known fungal secondary metabolism gene clusters. Columns indicate, in this order, the name of the metabolite; the pubmed ID of the paper where the cluster was described; the species in which the cluster was first described; and the complete reference of the paper.

Supplementary table 18: List of families with identified secondary metabolism gene clusters. The first column indicates the cluster name as seen also in Supplementary Table 2. Second column indicates the fine-grained evolutionary mechanism through which the cluster family evolved. The following columns indicate the putative compounds synthesized by the clusters. Multiple compounds indicate that either a single cluster is able to synthesize both compounds or that different clusters in the family are able to synthesize those compounds.

Supplementary table 19: List of families with a predicted function in secondary metabolism. 